\title{
Calibration of a high spectral resolution lidar using a Michelson interferometer, with data examples from ORACLES
}

\author{
S. P. Burton ${ }^{1 *}$, C.A. Hostetler ${ }^{1}$, A.L. COOK $^{1}$, J.W. HAir ${ }^{1}$, S.T. \\ Seaman ${ }^{1}$, S. SCOla ${ }^{1}$, D.B. HARPer ${ }^{1}$, J.A. SMith ${ }^{1}$, M.A. FenN ${ }^{2,1}$, R.A. \\ FerRare ${ }^{1}$, P.E. SAIDE ${ }^{3}$, E.V. CheMYAKIN ${ }^{2,1}$, D. MÜLleR ${ }^{4}$ \\ ${ }^{1}$ NASA Langley Research Center, Hampton, VA, 23681, USA \\ ${ }^{2}$ Science Systems and Applications, Inc., Hampton, VA, 23666, USA \\ ${ }^{3}$ Department of Atmospheric \& Oceanic Sciences, University of California, Los Angeles, CA, 90095, USA \\ ${ }^{4}$ University of Hertfordshire, Hatfield, Hertfordshire, AL10 9AB, UK \\ *Corresponding author: sharon.p.burton@nasa.gov
}

Received XX Month XXXX; revised XX Month, XXXX; accepted XX Month XXXX; posted XX Month XXXX (Doc. ID XXXXX); published XX Month XXXX

\begin{abstract}
The NASA Langley airborne 2nd generation High Spectral Resolution Lidar (HSRL-2) uses a density-tuned fieldwidened Michelson interferometer to implement the HSRL technique at $355 \mathrm{~nm}$. The Michelson interferometer optically separates the received backscattered light between two channels, one of which is dominated by molecular backscattering while the other contains most of the light backscattered by particles. This interferometer achieves high and stable contrast ratio, defined as the ratio of particulate backscatter signal received by the two channels. We show that a high and stable contrast ratio is critical for precise and accurate backscatter and extinction retrievals. Here we present retrieval equations that take into account the incomplete separation of particulate and molecular backscatter in the measurement channels. We also show how the accuracy of the contrast ratio assessment propagates to error in the optical properties. For both backscattering and extinction, larger errors are produced by underestimates of the contrast ratio (compared to overestimates), more extreme aerosol loading, and-- most critically-- smaller true contrast ratios. We show example results from HSRL-2 aboard the NASA ER-2 aircraft from the 2016 ORACLES field campaign in the southeast Atlantic off the coast of Africa during the biomass burning season. We include a case study where smoke aerosol in two adjacent altitude layers showed opposite differences in extinction- and backscatter-related Ångström exponents and a reversal of the lidar ratio spectral dependence, signatures which are shown to be consistent with a relatively modest difference in smoke particle size.

OCIS codes: (010.1110) Aerosols; (010.3640) Lidar; (010.0010); Atmospheric and oceanic optics, Remote sensing.
\end{abstract}

\section{Introduction}

Vertical profiling of aerosol by lidar contributes critically to scientific understanding of air quality, ocean ecosystems, Earth's radiation budget, and atmospheric processes and transport, including stratosphere-troposphere exchange and long-range transport of aerosols. Among different types of lidars, the Raman and High Spectral Resolution Lidar (HSRL) techniques provide vertically resolved aerosol backscatter and extinction independently without the need to assume the aerosol extinction-to-backscatter ratio or rely on a column optical depth constraint. Multi-wavelength lidars are especially useful for constraining particle size and enable a basic microphysical inversion [1, 2]. The $355 \mathrm{~nm}$ wavelength, in comparison to $532 \mathrm{~nm}$ and $1064 \mathrm{~nm}$, gives more sensitivity to smaller particles, which are relevant to studies of aerosol-cloud interactions, which in turn are critical for reducing uncertainty in climate change predictions.

This contribution is focused primarily on the NASA Langley airborne $2^{\text {nd }}$ generation High Spectral Resolution Lidar (HSRL-2), which makes three-wavelength lidar measurements of the atmosphere. Except for the addition of the $355 \mathrm{~nm}$ channels, HSRL-2 is essentially similar to the first NASA Langley airborne HSRL-1 instrument that has been fully described [3] and validated [4] previously. The HSRL technique is well suited to aircraft operations and in general has finer resolution and better daytime performance than the Raman technique. The HSRL technique is implemented with an iodine gas filter at $532 \mathrm{~nm}$ in HSRL-2, but implementation at $355 \mathrm{~nm}$ requires using an interferometer. The interferometer technique may also be more desirable in some applications because of greater throughput of light, which therefore requires less averaging for the same random error. Calibration and 
aerosol retrieval algorithms are more complex with the interferometer design, because of the imperfect separation between channels. Describing the calibration and retrieval methodology is the focus of this paper.

To review, the HSRL technique [5] is a method of measuring aerosol and cloud extinction and backscatter coefficients using a lidar with two detector channels. It is an improvement over the elastic backscatter lidar technique which uses a single channel that observes attenuated backscatter. The single-channel lidar equation is given by the following:

$$
\frac{P_{p}(r)}{g_{p}}=\frac{1}{r^{2}}\left[\beta_{m}(r)+\beta_{p}(r)\right] \exp \left\{-2 \int_{0}^{r}\left[\alpha_{m}\left(r^{\prime}\right)+\alpha_{p}\left(r^{\prime}\right)\right] d r^{\prime}\right\}
$$

where $P_{p}$ indicates the calibrated signal at the detector, which has had the electronic and daylight background signals subtracted. It is a function of $r$, which is the range (distance) between the lidar and the target atmospheric volume. The backscatter coefficient of particles (aerosol or optically thin clouds) at range $r$ is given by $\beta_{p}(r)$ and of air molecules is given by $\beta_{m}(r)$. The exponential term represents attenuation of the light on the two-way journey between the lidar and the scattering volume, where $\alpha_{p}$ is the particulate extinction coefficient and $\alpha_{m}$ is the molecular extinction coefficient. The coefficient $g_{p}$ is a gain factor which takes into account detector and receiver optical efficiencies.

The HSRL technique uses a second channel that is dominated by the molecular signal. This technique requires a precision filter. It has been implemented at $532 \mathrm{~nm}$ using an iodine gas filter. This filter blocks light at the transmitted wavelength from reaching the detector in the HSRL channel, while passing light at nearby wavelengths. The molecular backscatter return is spectrally broadened due to Cabannes scattering [6], while the scattered light from the heavier aerosol and cloud particles remains at the transmitted wavelength. Therefore, the filtered HSRL channel suppresses scattered light from particulate matter and allows scattered light from air molecules, as represented in the following equation,

$$
\frac{P_{m}(r)}{g_{m}}=\frac{1}{r^{2}} \beta_{m}(r) \exp \left\{-2 \int_{0}^{r}\left[\alpha_{m}\left(r^{\prime}\right)+\alpha_{p}\left(r^{\prime}\right)\right] d r^{\prime}\right\}
$$

where $P_{m}$ indicates the background-subtracted signal at the detector in the molecular-dominated channel, and $g_{m}$ is the gain factor for this channel. For the NASA Langley airborne HSRL-2 instrument, rotational Raman scattering side bands are suppressed in both channels due to the narrow spectral bandpass [7] and so are not discussed here.

The existence of the filtered HSRL channel is the key to the retrieval of true backscatter and extinction coefficients. Since molecular scattering and extinction coefficients can be calculated accurately using a measurement of atmospheric density or a good meteorological model, the $\beta_{m}(r)$ and $\alpha_{m}(r)$ terms are known. Therefore, the moleculardominated channel is a measure of the attenuation. Dividing Eq. (1) by Eq. (2) cancels out the attenuation term and range-squared dependence. This ratio directly provides the true backscatter coefficient, unaffected by attenuation. The particulate extinction $\alpha_{p}(r)$ is easily obtained from the molecular-dominated channel, since it is the only unknown variable in Eq. (2).

$$
\begin{gathered}
\beta_{p}(r)=\left(\frac{P_{p}(r)}{P_{m}(r)} \frac{g_{m}}{g_{p}}-1\right) \beta_{m}(r) \\
\alpha_{p}(r)=-\frac{1}{2} \frac{d}{d r} \ln \left(\frac{r^{2} P_{m}(r)}{g_{m} \beta_{m}(r)}\right)-\alpha_{m}(r)
\end{gathered}
$$

These equations hold for the case of a perfect filter. (For simplicity in this introduction, Eq. (4) is given for a two-channel system. For an instrument with a polarization channel, the equation must take all three channels into account [3]).
While the iodine filter technique is used at $532 \mathrm{~nm}$ in the NASA Langley airborne High Spectral Resolution Lidar instruments, at $355 \mathrm{~nm}$ there is no equivalent gas-filter design available. Instead, various designs of interferometers have been used for the implementation of the high spectral resolution lidar technique at $355 \mathrm{~nm}$ in NASA Langley's airborne HSRL-2 and other airborne and spaceborne instrument designs [5, 8-10]. HSRL-2 uses a Michelson interferometer. It splits the incoming beam into two beams that travel different optical path lengths and then are superposed with different phase to create constructive and destructive interference in the two HSRL optical channels.

Section 2 describes the Langley Michelson interferometer and how it is optimized for implementation of the HSRL technique. Section 3 describes the retrieval of aerosol backscatter and extinction from the channel returns. Section 4 assesses the requirement for accuracy of the characterization of the interferometer contrast ratio, to achieve desired accuracy in the particulate backscatter and extinction retrievals. Section 5 discusses how the interferometer contrast ratio has been characterized operationally during the ObseRvations of Aerosols above CLouds and their intEractionS (ORACLES) field campaign. Section 6 discusses some example retrieval results from that campaign that rely on this calibration.

\section{2. $355 \mathrm{~nm}$ Density-tuned Field-widened Michelson interferometer}

HSRL-2 includes three polarization-sensitive wavelengths, two of which employ the HSRL technique. At $355 \mathrm{~nm}$, an interferometer is used to implement the HSRL technique after the polarization split. The Langley interferometer is a density-tuned (sometimes called "pressuretuned") field-widened Michelson interferometer [11], see Figure 1. On entering the interferometer, the input beam is split by a 50/50 beam splitter and the two component beams travel through a solid glass arm and a sealed air arm, reflect off mirrors, and recombine with different phases. The different densities of the air and glass arms result in the necessary difference in optical path length to create the phase shift. The interferometer is off-axis compared to the incoming beam, allowing for both output channels to be captured by photodetectors. The interferometer is tuned to create destructive interference at the transmitted frequency for one of the channels, thereby blocking most of the particulate signal in that channel, the molecular-dominated channel. The particulate-dominated channel has constructive interference at the transmitted frequency, and therefore contains most of the particulate signal (when present).

The particulate signal is not completely rejected from the moleculardominated channel, as it is with the iodine filter technique. That channel therefore also includes some residual particulate backscatter, an effect sometimes referred to as Rayleigh-Mie cross-talk [e.g. 12]. Likewise, the particulate-dominated signal will include some fraction of the molecular backscattered light. This is represented by the following equations (after [5]).

$$
\begin{aligned}
& \frac{P_{m}(r)}{g_{m}}=\frac{1}{r^{2}}\left[A \beta_{m}^{\|}(r)+B \beta_{p}^{\|}(r)\right] T(r)^{2} \\
& \frac{P_{p}(r)}{g_{p}}=\frac{1}{r^{2}}\left[C \beta_{m}^{\|}(r)+D \beta_{p}^{\|}(r)\right] T(r)^{2}
\end{aligned}
$$

Since the implementation in the HSRL-2 instrument uses the interferometer only on the component of the backscattered light that is co-polarized with respect to the transmitted beam, we have added a superscript to the backscatter terms. Eqs. (5) and (6) are a more generalized form of Eqs. (1) and (2), with the exponential two-way transmittance term simplified and represented as $T(r)^{2}$. The coefficients $A, B, C$, and $D$ indicate that some fraction of the backscattered light from both particulate and molecular sources is detected in each of the two 
detector channels. In our formulation $A+C=B+D=1$. Any overall losses are included in the channel gains $g_{m}$ and $g_{p}$. Furthermore, if the broadened molecular signal is divided evenly between the two channels, $C=A$. In the HSRL-2 instrument, to ensure as even a split as possible, differential losses in the beam splitter were minimized (split ratio 50.7:49.3), and the free spectral range (FSR) of the Michelson interferometer is optimized to distribute the molecular signal evenly at the operational temperatures (FSR $=2.0 \mathrm{GHz}$ ). Given the conditions $A+$ $C=B+D=1$ and $A \approx C \approx 0.5$, there is only one unknown parameter among the four coefficients $A, B, C$ and $D$, and that is the ratio $D / B$. The ratio $D / B$, or contrast ratio, is an important indicator of interferometer performance since it specifies the ability to distinguish particulate and molecular backscattered light. Note that this ratio is not the same as the "fringe contrast" which is often used in interferometry. For an ideal interferometer, $D / B$ would be infinite, but mirror surface roughness, misalignment, tilt error, and imperfect tuning to the frequency of maximum constructive/destructive interference have the potential to reduce $D$ and increase $B$ such that real interferometers have finite contrast ratio.

Since the coefficients $A, B, C$, and $D$ are required in Eq. (5) and (6) for the retrieval of particulate backscatter and extinction coefficients, the accuracy of the retrieved quantities depends on characterizing the contrast ratio accurately. Errors in the assessed contrast ratio lead to systematic errors in the retrieved backscatter and extinction. As we will show in Section 4, the impact of this error source is minimized for large values of the contrast ratio.

Not only the accuracy, but also the precision of the retrieval depends on the magnitude of the contrast ratio. The higher the contrast ratio, the better the discrimination of particulate and molecular backscatter returns. The upper limit of infinity represents a perfect filter, which the $532 \mathrm{~nm}$ iodine filter approximates well. In the lower limit, if the contrast ratio is 1 (both particulate and molecular backscatter are equally split between the two channels), there is no ability to discriminate particulate and molecular backscatter sources, and therefore no way to determine the particulate extinction or correct the particulate backscatter coefficient for attenuation.

The NASA Langley density-tuned field-widened Michelson Interferometer is specially designed to achieve the high and stable contrast ratios that are important for the HSRL retrieval at $355 \mathrm{~nm}$. In this version of the interferometer, both mirrors are fixed. However, variations in temperature cause variability in both the optical and physical lengths of the arms due to thermal expansion and slight changes in the refractive index of the glass in the solid arm. To lock the interferometer to its optimum performance in flight, the air density inside the air arm is adjusted using a bellows to pump air in or out (see Figure 1), thereby fine-tuning the index of refraction and, hence, optical path length of the air arm. The interferometer is continuously and automatically locked to the target frequency ( $355 \mathrm{~nm}$ ) by means of an auxiliary beam from the seed laser. This beam is inserted into the interferometer in the shadow of the telescope secondary mirror which therefore remains spatially separated from the atmospheric signal while passing through the interferometer to a separate detector.

Other sources of error that can compromise optimum contrast performance include mirror tilt and wavefront error. Therefore the performance was optimized at the operating temperature by two rounds of corrective polishing $[11,13]$. In flight, the instrument is enclosed in an environmentally controlled box, and the temperature of the interferometer housing is carefully controlled. In addition, a thermal gradient is applied that can be adjusted in flight to counteract any residual relative tilt between the solid and air arm mirrors.

The optimization of the interferometer design and the operation in a thermally controlled environment with a controlled thermal gradient has resulted in very high contrast ratios of 35-60 when operating HSRL-
2 aboard the high-flying NASA ER-2 during the ORACLES 2016 field campaign, as shown in Figure 2. The continuous adjustment of the density inside the air arm allows the performance to be very stable after an initial temperature stabilization period in the first 30 to 60 minutes of a flight.

\section{Retrieval of aerosol backscatter and extinction coefficients from interferometric HSRL technique}

To get the particulate extinction coefficient, we multiply Eq. (5) by $D$ and Eq. (6) by $B$ and subtract to remove the $\beta_{p}$ term.

$$
\begin{aligned}
& T(r)^{2}=\exp \left\{-2 \int_{0}^{r}\left[\alpha_{m}\left(r^{\prime}\right)+\alpha_{p}\left(r^{\prime}\right)\right] d r^{\prime}\right\} \\
& =\frac{1}{(A D-B C)} \frac{r^{2}}{\beta_{m}^{\|}(r)}\left(D \frac{P_{m}(r)}{g_{m}}-B \frac{P_{p}(r)}{g_{p}}\right)
\end{aligned}
$$

Taking the natural logarithm of both sides and then the derivative yields

$$
\alpha_{p}(r)=-\frac{1}{2} \frac{\partial}{\partial r} \ln \left\{\frac{r^{2}}{\beta_{m}^{\|}(r)}\left(D \frac{P_{m}(r)}{g_{m}}-B \frac{P_{p}(r)}{g_{p}}\right)\right\}-\alpha_{m}(r)
$$

In processing, the $\beta_{m}(r)$ and $\alpha_{m}(r)$ terms are calculated from the molecular density profile from the Modern-Era Retrospective Analysis for Research and Applications, version 2 (MERRA2) reanalysis of the Goddard Earth Observing System model (GEOS-5) [14] at the time and location of the lidar measurements. The derivative is calculated numerically from minimally smoothed signal profiles.

Recall that the HSRL channel signals $P_{m}$ and $P_{p}$ are split from the component of the returned signal that is co-polarized with the transmitted signal. To calculate the particulate backscatter, the crosspolarized signal must also be included.

$$
\frac{P_{\perp}(r)}{g_{\perp}}=\frac{1}{r^{2}}\left[\beta_{m}^{\perp}(r)+\beta_{p}^{\perp}(r)\right] T(r)^{2}
$$

The total molecular backscatter coefficient and the total particulate backscatter coefficient are given by

$$
\begin{gathered}
\beta_{m}(r)=\beta_{m}^{\perp}(r)+\beta_{m}^{\|}(r) \\
\beta_{p}(r)=\beta_{p}^{\perp}(r)+\beta_{p}^{\|}(r)
\end{gathered}
$$

Combining Eq. (5), (6), and (9) we arrive at the following expression for the total particulate backscatter:

$$
\begin{gathered}
\beta_{p}(r)=\frac{\beta_{m}(r)}{1+\delta_{m}} \times \\
\left\{\left(\frac{A \frac{g_{m}}{g_{p}} \frac{P_{p}(r)}{P_{m}(r)}-C+(A D-B C) \frac{g_{m}}{g_{\perp}} \frac{P_{\perp}(r)}{P_{m}(r)}}{D-B \frac{g_{m}}{g_{p}} \frac{P_{p}(r)}{P_{m}(r)}}\right)-\delta_{m}\right\}
\end{gathered}
$$

The term $\delta_{m}$ represents the molecular depolarization, defined as the perpendicular component of molecular backscatter coefficient divided by the parallel component. The value is obtained theoretically [15].

As discussed above, the coefficients $A$ and $C$ are equal to 0.5 for the HSRL-2 instrument. Section 5 will describe how the contrast ratio $D / B$ is determined. Once it is determined, the variables $B$ and $D$ are calculated from the contrast ratio by

$$
\begin{aligned}
& B=\frac{1}{D / B+1} \\
& D=\frac{D / B}{D / B+1}
\end{aligned}
$$

\section{Requirement on contrast ratio accuracy}

For an optimally precise and accurate retrieval of aerosol backscatter and extinction, two conditions must be met: the contrast ratio must be 
high and it must be accurately characterized. The design of the Langley interferometer results in high contrast ratios and a high degree of stability that makes characterization of the contrast ratio possible. In this section, we discuss how accurate the characterization needs to be to achieve optimal accuracy in backscatter and extinction retrievals. In the next section, we describe how the characterization of the contrast ratio has been performed for a recent airborne mission.

Figure 3 shows the sensitivity of the backscatter coefficient to errors in the characterization of the contrast ratio, following from numerical differentiation of Eq. (12). In general, underestimates of the contrast ratio result in overestimates of the backscatter coefficient, and vice versa, with the error being smaller for overestimates than for underestimates. The consequence of a given error in contrast ratio decreases as the true contrast ratio increases. That is, better interferometer performance results in less sensitivity to the accuracy of the contrast ratio characterization. The size of the error also grows with the total scattering ratio (TSR: the ratio of aerosol plus molecular backscattering to molecular backscattering).

For a nominal contrast ratio of 40 , backscatter uncertainty of $5 \%$ or less (to one standard deviation) can be achieved if the contrast ratio is known to within \pm 8 (one standard deviation), for aerosol loading of TSR $<4$ which encompasses most typical aerosol scenarios. For very heavy loading, the uncertainty will be greater. In order to achieve backscatter uncertainty of $5 \%$ for a loading of TSR $=11$, which may be seen in heavily polluted regions in Asia, for instance, it would be necessary to determine the contrast ratio to an accuracy of \pm 3 .

An error in the inferred contrast ratio can also produce an error in the extinction coefficient. In this case, besides the dependencies on true contrast ratio and on overall aerosol loading (represented by total scattering ratio), there is an additional dependence of the error on the gradient of the aerosol extinction profile. These dependencies are illustrated in Figure 4. The simulated profile in Figure 4a is constructed to have smoothly varying gradients to illustrate the dependence of the error propagation on extinction gradient. The largest gradients are at the bottom and top of the simulated aerosol layer. Panel $4 \mathrm{~b}$ shows the partial derivative of the extinction with respect to the contrast ratio, or in other words, the error in extinction coefficient due to a unit error in contrast ratio. This propagation factor is largest where the extinction gradient is largest. This means that errors in the inferred contrast ratio will primarily be exposed at the edges of aerosol and cloud layers. Finally, Figure 4c shows the absolute error in extinction for various errors in the inferred contrast ratio for the same cases that were illustrated in Figure 3, but now specifically for an extinction gradient of $0.5 \mathrm{~km}^{-1}$ per $\mathrm{km}$ (the bottom edge of the simulated aerosol layer). As with the error in backscatter, the error in extinction is minimized for larger values of the true contrast ratio and is less for overestimates of the contrast ratio than for underestimates. The dependence of the absolute extinction error on TSR (likewise on the absolute value of the extinction) is weak. Given this, the percent error in extinction can be significant if the absolute value is small. To summarize: for a nominal true contrast ratio of 40 and TSR $<4$, an overestimate (underestimate) of the contrast ratio by 10 would produce an extinction error of 0.002 $\mathrm{km}^{-1}\left(0.005 \mathrm{~km}^{-1}\right)$ for a gradient of this size.

Since the extinction error is sensitive to the gradient of the aerosol profile but the backscatter error is not, there is also a strong sensitivity in the lidar ratio, at layer edges. Since the lidar ratio generally has less variability within a given aerosol layer, lidar ratio errors at the edges of layers can be more obvious and can give a qualitative check of the contrast ratio.

Figure 5 illustrates the errors in profiles of aerosol backscatter, aerosol extinction and lidar ratio resulting from errors in the contrast ratio, using a measured profile of biomass burning aerosol during ORACLES and a large range of assumed contrast ratios.

\section{Operational contrast ratio assessment}

One method to determine the contrast ratio is to inject single frequency light at the transmitted laser frequency into both HSRL optical channels. If the detector shutters are closed and no atmospheric signal is present, only the $B$ and $D$ terms of Eqs. (5) and (6) are non-zero. This method has the advantage that it can be used for real-time assessment and optimization. However, this injected signal may not illuminate the detector with the same profile of intensity over its active area, as compared to atmospheric observations. If the detector exhibits differences in responsivity across its active area as many detectors do, the differences in responsivity will create an error in the contrast ratio estimated via this method.

A more accurate option for atmospheric data processing, and simpler to implement in terms of hardware, is to use an atmospheric target. Accordingly, we can infer the contrast ratio using the lidar return from an opaque target such as water cloud, as long as this signal does not saturate the detector or associated electronics.

By combining Eqs. (5) and (6) for the returns from an opaque target, we get the following expression for the contrastratio, evaluated at $r_{c}$, the range of the cloud or other target.

$$
\frac{D}{B}=\frac{P_{p}\left(r_{c}\right) r_{c}{ }^{2} / g_{p}-C \beta_{m}^{\|}\left(r_{c}\right) T\left(r_{c}\right)^{2}}{P_{m}\left(r_{c}\right) r_{c}{ }^{2} / g_{m}-A \beta_{m}^{\|}\left(r_{c}\right) T\left(r_{c}\right)^{2}}
$$

Note that the second terms in both the numerator and denominator on the right side of Eq. (15) are small compared to the first terms in the case of an opaque target; therefore, Eq. (15) can be thought of as approximately the ratio of the calibrated signals in the two channels from the top surface of the opaque target.

Figure 6 shows the calibrated signals times range squared, for the $355 \mathrm{~nm}$ particulate-dominated and molecular-dominated channels, shown as a function of range from the instrument on board the NASA ER-2 aircraft. The lidar profile was measured on 18 September 2016 in the southeast Atlantic. In the particulate-dominated channel, the observation shows a moderately thick smoke plume with layer optical depth of about 0.3 at $355 \mathrm{~nm}$ spread between 15.5 and $18.2 \mathrm{~km}$ range from the aircraft. However, the lidar return from the opaque cloud surface at $18.7 \mathrm{~km}$ dominates this aerosol signal by a factor of more than 100. In the molecular-dominated channel, the cloud spike is partially suppressed by the destructive interference in the interferometer, but is nevertheless three to seven times larger than the signal from the molecular atmosphere in that channel as well.

Figure 7 shows backscattered signal data from 16 September 2016 from the top of opaque clouds at two altitude levels, color coded by time. Each data point is an integrated return over a few bins comprising the cloud spike. Calibrated data from the aerosol-dominated channel is shown on the x-axis, and from the molecular channel on the y-axis. The data are notably linear, although there are regime changes throughout the flight in which the slope or intercept changes over time.

In Eq. (6), which represents the signal in the aerosol-dominated channel, the term $D \beta_{p}$ is very much larger than $C \beta_{m}$ for an opaque target, given any reasonable value of the contrast ratio. However, this is not the case for the terms in Eq. (5). While $A \beta_{m}$ is smaller than $B \beta_{p}$ for an opaque target, it cannot be entirely neglected and its strength, relative to the aerosol term, is greater for larger contrast ratios. The linear relationship in Figure 7 can therefore be explained by rearranging the terms in Eq. (15) and neglecting the term $C \beta_{m}$ term while retaining the $A \beta_{m}$ term.

$$
\frac{P_{m} r^{2}}{g_{m}} \approx \frac{B}{D} \frac{P_{p} r^{2}}{g_{p}}+A \beta_{m}^{\|} T^{2}
$$

Eq. (16) is in the form of a line with the slope giving the inverse of the contrast ratio and the intercept being proportional to the attenuated molecular backscatter near the cloud top. Changes in the slope in Figure 7 represent true changes in the interferometer contrast ratio, due to, for 
example, temperature changes in the interferometer. Changes in the intercept indicate differences in attenuated molecular backscattering near the cloud top, due to differences either in cloud-top height or in the intervening attenuation.

Using Eq. (16) for estimation of the contrast ratio requires a linear fit, which requires a discrete set of data with a relatively constant contrast ratio over some length of time. With the advanced interferometer design described in Section 2, the contrast ratio is sufficiently stable to achieve this requirement.

Four subsets of up to an hour from the data from 16 September 2016 are shown again in Figure 8. There is noticeable consistency in the slope (contrast ratio) over the course of the flight, but the intercept exhibits both gradual and discrete changes throughout the flight. Changes in the intercept indicate that the attenuated molecular backscatter term $A \beta_{m} T^{2}$ is changing. Obvious differences in intercept occur during time segments where there are both low-level and mid-level clouds (panels a and c), which can be seen also in the HSRL-2 backscatter curtain shown in Figure 9. For most of the flight, the low-level clouds are observed through a thick smoke layer that decreases the $T^{2}$ term, so overall the intercept of the line defined by low clouds is smaller in these segments than the intercept from the mid-level cloud segments. For portions of the flight where the low-level clouds are relatively unobscured by overlying aerosol, such as after $13 \mathrm{UT}$, the intercept becomes larger again. There is also a larger dynamic range for these relatively unattenuated signals corresponding to either low clouds with little aerosol above, or mid-level clouds at the top of the aerosol layer.

In short, data aggregation is necessary because the contrast ratio is estimated mostaccurately using a linear fit to a discrete segment of data. On the other hand, there is a tradeoff in that too much data aggregation risks combining data with different true contrast ratios. There is also a risk of introducing error into the fit by combining data that have different intercepts. Operationally, for HSRL-2 processing, we group the data into 5-30 minutes segments, and separate signals from cloud tops in different height regimes. Fits are more accurate for segments with a large dynamic range compared to the noise level. Viewed from the NASA ER-2, low cloud data are far enough away from the aircraft that the dynamic range of the molecular signal is often relatively small, so a time interval of 30 minutes was used in final processing of the publically archived ORACLES data from the NASA ER-2. For flights conducted at the much lower cruising altitude of the NASA P-3B, less data aggregation is necessary. We further increase the linearity of these data segments by making a first rough estimation of the attenuated molecular term, $A \beta_{m} T^{2}$ or $C \beta_{m} T^{2}$ (recall $A=C$ by design, see Section 2), on a profile-byprofile basis, before performing the linear fit. This estimate is simply the value of the attenuated molecular signal from the range bins just above the cloud signal spike. It is not critical for this estimated intercept to be very accurate in order to retrieve the contrast ratio from the slope. Random errors in the estimated intercept will merely have the effect of broadening the line, while systematic errors are of no concern since they would have little impact on the slope. The procedure in use for the HSRL-2 processing of ORACLES data is summarized in Table 1.

\section{A. Gain ratio assessment}

We note that Eqs. (1)-(16) represent a calibrated lidar signal with all necessary correction factors applied except the interferometer crosstalk coefficients, which we address in this paper. The detector gain factors that reflect the optical efficiency and electronic gains of the detector channels are briefly considered in more detail here, since they also depend on the interferometer coefficients. As described previously [3], the iodine channel (532 $\mathrm{nm}$ ) is calibrated straightforwardly and accurately by mechanically translating the iodine cell out of the beam path to equalize the signals in the total backscattering channel and the molecular-only channel. Similar to the $1064 \mathrm{~nm}$ channel calibration [3], the $355 \mathrm{~nm}$ channel calibration makes use of the accurate $532 \mathrm{~nm}$ channel calibration. The calibration is done in a relatively aerosol-free region of the free troposphere where the $532 \mathrm{~nm}$ TSR does not exceed about 1.05. Although the aerosol amount in the calibration region is small, it is not neglected. The true aerosol backscatter coefficient obtained at $532 \mathrm{~nm}$ is converted to $355 \mathrm{~nm}$ by assuming the "background" aerosol high in the free troposphere has a color ratio of 0.4 , which can also be expressed as a backscatter-related Ångström exponent of 1.32. The $355 \mathrm{~nm}$ backscatter coefficient also depends on the interferometer coefficients $A, B, C$ and $D$, as discussed in Section 3 . Therefore, the determination of the aerosol-to-molecular channel gain ratio and the determination of the contrast ratio $D / B$ are related nonlinearly and a first-guess contrast ratio $D / B$ is required in the gain ratio calibration step.

Figure 10 shows the error in the gain ratio between the aerosoldominated and molecular-dominated $355 \mathrm{~nm}$ channels for various contrast ratios given that the contrast ratio is assumed to be 20 in the calibration step, for realistic values of the gain ratio and the aerosol to molecular backscatter ratio. For any value of the contrast ratio larger than approximately 10 , the error is less than half a percent in the gain ratio. Since the error is small compared to other uncertainties in the gain ratio $[3,7]$, we do not require an iteration of the gain ratio calculation using the updated contrast ratio estimate.

Although the effect of error in the contrast ratio on error in the gain ratio is negligible, the opposite effect should not be ignored. That is, an error in the assessment of the gain ratio will cause a nearly proportional error in the contrast ratio, which propagates to an additional error in the backscatter and extinction retrieval, as we have already shown. In a previous work [7], we estimate the gain ratio uncertainty in the $355 \mathrm{~nm}$ channel at 3\%. This has a nearly proportional effect on the uncertainty in the contrast ratio (ignoring the intercept term), so for a contrast ratio of 40 , this adds an additional uncertainty of 1.2 in contrast ratio units, but this in turn has only a small effect on the backscatter and extinction uncertainty for typical aerosol loading, as discussed above.

Note that other gain ratio calibrations including the depolarization gain calibration are discussed elsewhere by $[3,7]$ and are the same for the $355 \mathrm{~nm}$ channel as the $532 \mathrm{~nm}$ channel.

\section{Data examples}

\section{A. Case Study 1, 18 September 2016}

By design, the ORACLES campaign [16] observed African biomass burning over the southeastern Atlantic over a nearly continuous stratus cloud deck. Figure 11 shows a profile averaged over 12 minutes from 11:02 to 11:14 UT on 18 September 2016 that includes the same data shown in Figure 5 and Figure 6 . The segment is $136 \mathrm{~km}$ along the $11.5 \mathrm{E}$ meridian between approximately $11.0^{\circ}$ and $12.2^{\circ} \mathrm{S}$, approximately 240 $\mathrm{km}$ off the coast of Angola. The backscatter and extinction show significantamounts of aerosol from cloud top (at approximately $0.9 \mathrm{~km}$ ) up to $5.3 \mathrm{~km}$, but with a large enhancement of approximately a factor of 2 or greater in the layer above $3.5 \mathrm{~km}$ compared to the layer below that level. The total above-cloud aerosol optical thickness for the smoke at this time is $0.38(532 \mathrm{~nm})$ and $0.63(355 \mathrm{~nm})$. Particulate depolarization is small above $2 \mathrm{~km}$, consistent with many previously published values for smoke-dominated airmasses [e.g. 17, 18-20] although there may also be a small amount of dust. Below $2 \mathrm{~km}$ there is less smoke, but the particulate depolarization ratios increase above $10 \%$, reflecting a larger (though still small) proportion of dust. The lidar ratios generally vary between $58 \mathrm{sr}$ and $76 \mathrm{sr}(532 \mathrm{~nm})$ and $65 \mathrm{sr}$ to $78 \mathrm{sr}(355 \mathrm{~nm})$, between 2 and $5 \mathrm{~km}$. The $532 \mathrm{~nm}$ value is consistent with most reported aged smoke values [e.g. 20, 21, 22 and references therein] and African biomass burning aerosol specifically [23-26]. Reported smoke lidar ratio values at $355 \mathrm{~nm}$ vary widely, even for African biomass burning 
smoke specifically. Values as low as of $40-50 \mathrm{sr}$ at $355 \mathrm{~nm}$ were reported for African smoke transported to the Amazon [23], while high values of $92 \pm 10 \mathrm{sr}$ were reported for a sample of elevated layers observed in South Africa [25]. Values in Figure 11 fall between these extremes and agree with the $355 \mathrm{~nm}$ lidar ratios reported by [26] and values for smoke retrieved from a dust-smoke mixture (by parameterizing some dust characteristics) [24]. Lidar ratios in Figure 11 decrease in the lowest altitudes near the cloud top, consistent with the increased dust mixing ratio below $2 \mathrm{~km}$. There is very little difference in the lidar ratio profiles between the two channels, and the ratio of the two channels ( $355 \mathrm{~nm} / 532 \mathrm{~nm}$ ) is $1.1 \pm 0.3$ (standard deviation of the selected data) between 2 and $5 \mathrm{~km}$, which is also consistent with previously reported values for smoke a few days old $[21,27]$.

In the upper part of the smoke layer, the extinction Ångström exponent is generally around $1.4 \pm 0.5$ (standard deviation). The backscatter related Ångström exponent is $1.3 \pm 0.4$ between 2 and $5 \mathrm{~km}$. (There is more variability in the lower part of the smoke layer because of reduced lidar return power due to the large amount of light extinction in the upper part of the layer.) These values agree within error bars with the values reported for South African observations by multi-wavelength Raman lidar [25]. In general, reported lidar measurements of both Ångström exponents for African biomass burning smoke vary widely with some values of extinction Ångström exponent as low as zero [23] and of backscatter Ångström exponent near 0.4 [26]; these two cited cases may include some dust mixed with the smoke. The extinction Ångström exponent is sensitive to particle size, with larger values indicating smaller particles [28], while the backscatter Ångström exponent has a complex sensitivity to particle size and also refractive index [29]. In short, lidar measurements of African smoke aerosol are sparse, but our observation falls within the ranges reported by other researchers.

Despite the paucity of previously reported lidar measurements of African biomass burning aerosol to compare with, we have high confidence in the accuracy of the reported measurements. The assessment of the contrast ratio, via the methodology presented in Section 5 , suggests that the contrast ratio is known to within about \pm 5 (unitless) plus the error of $\sim 3 \%(=1.2-1.5$ for contrast ratio of $40-50)$ due to uncertainty in the gain ratio between channels, discussed above. According to the error analysis of Section 4, a contrast ratio uncertainty of \pm 6.5 contributes only a very small amount to the uncertainties in the backscatter and extinction, as represented by gray shaded areas in Figure 11, which are small enough that they are barely discernable.

\section{B. Case Study 2, 20 September 2016}

A primary advantage conferred by making accurate HSRL measurements at $355 \mathrm{~nm}$ in addition to $532 \mathrm{~nm}$ is the additional information it provides related to aerosol properties, for qualitative interpretation $[7,20]$ and full microphysical retrievals $[1,2]$. Compared to Figure 11, the aerosol intensive parameters are less homogeneous in our second example, illustrated in Figure 12, which shows average profiles from a 12 minute segment of data from 09:54-10:06 UT on 20 September 2016, about $180 \mathrm{~km}$ off the coast of Angola at a location about $270 \mathrm{~km}$ away from Case Study 1 . Here, the aerosol intensive parameters reflect distinct differences in aerosol properties below and above about $4.4 \mathrm{~km}$. In particular, note systematic changes in the particle depolarization ratio, the extinction Ångström exponent and the backscatter Ångström exponent above vs. below $4.4 \mathrm{~km}$. Also note that the spectral dependence of the lidar ratio reverses, with the lidar ratio at $532 \mathrm{~nm}$ exceeding $355 \mathrm{~nm}$ above $4.4 \mathrm{~km}$, and the lidar ratio at $355 \mathrm{~nm}$ significantly exceeding the value at $532 \mathrm{~nm}$ below $4.4 \mathrm{~km}$.

Although there is a difference in particulate depolarization between the two layers, even the larger values are fairly low, $7 \% \pm 2 \%$ at $355 \mathrm{~nm}$ and $5.2 \% \pm 0.9 \%$ at $532 \mathrm{~nm}$. This argues that smoke dominates the airmass for the profile shown. Although there may again be trace amounts of dust present, note that in this case, in contrast to the case on 18 September 2016, the $355 \mathrm{~nm}$ particulate depolarization exceeds the $532 \mathrm{~nm}$ particulate depolarization ratio. This strongly suggests the presence of non-spherical smoke particles rather than coarse-mode dust [7]. (We note that at other locations on the same flight especially at low altitudes, not shown, we infer more dust from HSRL-2 observations).

Model forecasts, started each day from the previous day's analysis, were generated using the Weather Research and Forecasting (WRF) model configured with cloud microphysics that include parameterization of aerosol activation as cloud condensation and ice nuclei $[30,31]$. These were used for flight planning during ORACLES 2016 and they show good agreement with observed smoke layer locations and magnitudes. The model forecast of aerosol extinction for the case study of 20 September 2016 is shown in Figure 12 for comparison. These forecasts included tracers tagged to each day of smoke emissions, which provide a distribution of smoke age from which the mean age is computed. The model suggests that the upper and lower layers on 20 September 2016 in Figure 12 are different airmasses with different histories and ages, and that neither layer is younger than a few days. This is interesting in view of the spectral relationship of the lidar ratios in these two layers. Above the transition, $355 \mathrm{~nm}$ lidar ratio is $67 \pm 2$, smaller than $532 \mathrm{~nm}$ lidar ratio of $77 \pm 7$, giving a spectral ratio of $0.88 \pm 0.10$. Below the transition altitude, the wavelength dependence is reversed $(80 \pm 7$ at $355 \mathrm{~nm}$ and $58 \pm 5$ at 532 $\mathrm{nm}$ ) and the ratio of lidar ratios is $1.42 \pm 0.37$. It has been previously [27] theorized, from six case studies of smoke transported to Romania, that the spectral ratio of lidar ratios for smoke is an indicator of smoke age and that very fresh smoke (hours old) has a ratio of lidar ratios $(355 / 532 \mathrm{~nm})$ much larger than $1(=1.6$ for their fresh smoke case study), but the ratio quickly drops below 1 for aged smoke.

A primary driver of changes in optical properties of smoke with aging is increases in particle size due to condensation and coagulation [32]. In Figure 13, we illustrate with simple Mie calculations using code from Bohren and Huffman [33] that a difference in particle size with no other difference in microphysical properties is sufficient to explain differences in the spectral dependence of the lidar ratio, the extinction coefficient and the backscatter coefficient as seen in our ORACLES example in Figure 12. This is demonstrated using a very simple particle model with monomodal log-normal particle size distribution having variable effective radius. For this illustration, the effective variance is set to 0.195 and the complex refractive index is 1.49-i0.013 (spectrally invariant). For these specifications, there is a transition point at effective radius = $0.15 \mu \mathrm{m}$, such that for smaller values the $355 \mathrm{~nm}$ lidar ratio exceeds that at $532 \mathrm{~nm}$ and for effective radius values greater than $0.15 \mu \mathrm{m}$, the spectral relationship of the lidar ratios reverses. The particle size at which this transition occurs changes with refractive index but there exists such a threshold for a wide range of refractive indices [29, Figure 2]. Also note that, in the same regime of particle sizes near $0.15 \mu \mathrm{m}$ the extinction Ångström exponent decreases and the backscatter Ångström exponent $(355 / 532 \mathrm{~nm})$ increases as particle size increases, all in accordance with the relationships shown in the two layers in Figure 12. This simplified example illustrates that there exists a regime where differences in size alone can explain the relationships seen in the 20 September 2016 case study. Similar differences in particle size may in some cases be explained by smoke particle aging as in the case studies of [27], and aging may also affect absorption properties of smoke, but aging is not necessary to explain changes in particle size.

Humidification can also drive particle growth, and consequently lidar optical properties [e.g. 34]. Also shown in Figure 12 is a profile of relative humidity interpolated from the WRF model to the same location and time. The difference in properties between the layers is 
accompanied by an increase in the relative humidity. However, we again note that the two layers are different airmasses with different histories, so it is possible they have different size particles regardless of humidification. The difference in effective radius for these layers is confirmed by the HSRL-2 TiARA microphysical retrieval [35] results, which give an effective radius for the upper layer of approximately 0.13 and for the lower layer of approximately 0.10 .

\section{Summary and Discussion}

The high spectral resolution lidar technique is a way of accurately retrieving aerosol backscatter and extinction using two channels, one of which filters out the backscattered particulate signal at the transmitted wavelength. When an interferometer is used to do the filtering, accurate and precise retrievals can be achieved if the molecular signal is split evenly between the two channels and the contrast ratio of the particulate signal between the channels is high and well characterized.

In this work, the backscatter and extinction retrieval equations are given with explicit dependence on the parameters characterizing the interferometer. We define the interferometer contrast ratio as the contribution of particulate scattering to the particulate-dominated channel divided by the contribution of particulate scattering to the molecular-dominated channel. We specifically discussed the operational assessment of the interferometer contrast ratio using linear fits to short segments of data from opaque cloud targets from the particulate-dominated and molecular-dominated channels. We show how the accuracy of the contrast ratio assessment propagates to error in the retrieval. Specifically, the backscatter coefficient will be biased high if the contrast ratio is underestimated and biased low to a lesser extent if the contrast ratio is overestimated. Larger values of the true instrument contrast ratio lessen the magnitude of the bias. For a nominal contrast ratio of 40 and aerosol total scattering ratio (ratio of the total of aerosol and molecular backscattering to the molecular-only backscattering) less than or equal to 4 , a backscatter coefficient uncertainty of $5 \%$ (one standard deviation) can be achieved by assessing the contrast ratio to within \pm 8 (one standard deviation). For the extinction coefficient, error in the contrast ratio assessment affects accuracy mainly at strong gradients (such as the edges of aerosol or cloud layers). For a gradient of $0.5 \mathrm{~km}^{-1}$ per $\mathrm{km}$ where the total scattering ratio is not more than 4 and the true contrast ratio is 40 , overestimating the contrast ratio by 10 (that is, assuming a value of 50), would produce an absolute error in the extinction at the gradient of $0.002 \mathrm{~km}^{-1}$. For both backscattering and extinction, larger errors are produced by underestimates of the contrast ratio (compared to overestimates), more extreme aerosol loading, and-- most criticallysmaller true contrast ratios. Therefore, large and accurately-assessed values of the contrast ratio permit optimally accurate and precise retrievals of particulate backscatter and extinction.

Accurate lidar retrievals at $355 \mathrm{~nm}$ allow for enhanced sensitivity to smaller particles, which are relevant to studies of aerosol-cloud interactions, which in turn are critical for reducing uncertainty in predictions of climate change. An accurate backscatter coefficient retrieval is also required for accurate particle depolarization retrievals, particularly at $355 \mathrm{~nm}$, which in turn facilitate measurements of smoke transport [7]. Multiwavelength lidars also enable a basic microphysical inversion $[2,29]$. With high speed detectors, the HSRL technique can also be used for ocean profiling [36] and the HSRL-2 instrument discussed here is currently being upgraded to enable ocean profiling capability at both $355 \mathrm{~nm}$ and $532 \mathrm{~nm}$. In ocean lidar profiling, $355 \mathrm{~nm}$ measurements provide greater penetration depth in waters with low concentrations of colored dissolved organic matter [36], the ability to discriminate phytoplankton absorption from color-dissolved organic matter, and information on the slope of the particle size distribution.
Here we described the density-tuned field-widened Michelson interferometer designed and implemented by NASA Langley for the 355 $\mathrm{nm}$ channel in the airborne HSRL-2 instrument. This instrument achieves high and stable contrast ratios in the range 35-60 with only gradual drift throughout the course of a typical 8-hour flight on board the NASA ER-2 aircraft. The stability of the contrast ratio allows for an accurate assessment of its value to be obtained via linear fitting. The high values of the contrast ratio are important for precision in the backscatter and extinction retrievals, and also for minimizing the biasing effect of errors in the contrast ratio assessment. Since there is no practical gas filter for $355 \mathrm{~nm}$ in analogy to the iodine filter used in the same instrument at $532 \mathrm{~nm}$, and since the Raman technique is impractical on an airborne platform due to long averaging times, this interferometer technology makes accurate HSRL retrievals at $355 \mathrm{~nm}$ possible on an airborne platform.

We showed example retrievals from HSRL-2 aboard the NASA ER-2 from the ORACLES field campaign in the southeast Atlantic off the coast of Africa during the biomass burning season. We specifically highlight aerosol intensive parameters that depend on the $355 \mathrm{~nm}$ extinction and backscatter retrieval, namely $355 \mathrm{~nm}$ lidar ratio, aerosol extinction Ångström exponent, and aerosol backscatter-related Ångström exponent. For these cases, those parameters fall within the ranges reported in literature for African biomass burning aerosol. We specifically discussed a case study on 20 September 2016 during the ORACLES field campaign in which smoke aerosol in two adjacent altitude layers, observed off the coast of Angola, showed opposite differences in extinction- and backscatter-related Ångström exponents and a reversal of the lidar ratio spectral dependence. These features were shown to be consistent with a relatively small increase in smoke particle size, for effective radii near $0.15 \mu \mathrm{m}$.

The airborne HSRL-2 instrument is a prototype of the lidar for the NASA Aerosol-Clouds-Ecosystem satellite mission, a response to the National Research Council Decadal Survey recommendation [37] recognizing the need for accurate, vertically resolved, global characterization of aerosol properties to constrain model estimates of aerosol-radiation interactions and aerosol-cloud interactions.

The new design of the interferometer described here is a critically enabling step for implementation on a space instrument. On a satellite instrument, an interferometer design might be desirable at $532 \mathrm{~nm}$ as well as $355 \mathrm{~nm}$, since more light is transmitted, compared to using a gas filter. Yet space flight requires a rugged design with stable performance that can be maintained for years with minimal remote control. The monolithic design demonstrated on HSRL-2 with fixed physical arm lengths is rugged enough for long-term space use. The ability to rapidly density-tune the interferometer has been critical for the application of this interferometer design for the aircraft environment, for which there are large excursions in the temperature. The ORACLES results presented here have demonstrated the success of the adaptive finetuning of the interferometer in this challenging airborne environment. Looking forward, our group has also designed and built a similar interferometer suitable for application on a spaceborne lidar. For the stable temperature environment on the receiver optical bench of a satellite instrument, density tuning will not be necessary. We do note that, from space, the dynamic range of signals from cloud tops or the earth's surface will be reduced, so the accumulation times to generate contrast ratio estimates will increase. However, test data and performance studies indicate that contrast ratios will remain stable over days to weeks, far exceeding anticipated accumulation times.

\section{Data availability}

The HSRL-2 ORACLES dataset describe here is available to the public at the following permanent NASA archive https://espoarchive.nasa.gov/archive/browse/oracles/ER2/HSRL2 


\section{Funding}

The NASA Aerosols-Clouds-Ecosystems (ACE) project and the NASA ORACLES project. High-performance computing support from Yellowstone (ark:/85065/d7wd3xhc) provided by NCAR's Computational and Information Systems Laboratory, sponsored by the National Science Foundation

\section{Acknowledgements}

We wish to thank Rich Hare and Terry Mack of the NASA Langley Engineering Directorate for their exceptional work on the HSRL-2 instrument, and the NASA Armstrong ER-2 pilots and crew for their dedication in support of our measurements.

\section{References}

1. D. Müller, U. Wandinger, and A. Ansmann, "Microphysical particle parameters from extinction and backscatter lidar data by inversion with regularization: theory," in Appl Optics, (1999), pp. 2346-2357.

2. I. Veselovskii, A. Kolgotin, V. Griaznov, D. Müller, U. Wandinger, and D. N. Whiteman, "Inversion with regularization for the retrieval of tropospheric aerosol parameters from multiwavelength lidar sounding," in Appl Optics, (2002), pp. 3685-3699.

3. J. W. Hair, C. A. Hostetler, A. L. Cook, D. B. Harper, R. A. Ferrare, T. L. Mack, W. Welch, L. R. Izquierdo, and F. E. Hovis, "Airborne High Spectral Resolution Lidar for profiling aerosol optical properties," in Appl Optics, (2008), pp. 6734-6752.

4. R. R. Rogers, J. W. Hair, C. A. Hostetler, R. A. Ferrare, M. D. Obland, A. L. Cook, D. B. Harper, S. P. Burton, Y. Shinozuka, C. S. McNaughton, A. D. Clarke, J. Redemann, P. B. Russell, J. M. Livingston, and L. I. Kleinman, "NASA LaRC airborne high spectral resolution lidar aerosol measurements during MILAGRO: observations and validation," in Atmos Chem Phys, (2009), pp. 4811-4826.

5. S. T. Shipley, D. H. Tracy, E. W. Eloranta, J. T. Trauger, J. T. Sroga, F. L. Roesler, and J. A. Weinman, "High Spectral Resolution Lidar to Measure Optical-Scattering Properties of Atmospheric Aerosols .1. Theory and Instrumentation," in Appl Optics, (1983), pp. 3716-3724.

6. C.-Y. She, "Spectral Structure of Laser Light Scattering Revisited: Bandwidths of Nonresonant Scattering Lidars," Appl. Opt. 40, 4875-4884 (2001).

7. S. P. Burton, J. W. Hair, M. Kahnert, R. A. Ferrare, C. A. Hostetler, A. L. Cook, D. B. Harper, T. A. Berkoff, S. T. Seaman, J. E. Collins, M. A. Fenn, and R. R. Rogers, "Observations of the spectral dependence of linear particle depolarization ratio of aerosols using NASA Langley airborne High Spectral Resolution Lidar," in Atmos. Chem. Phys., (Copernicus Publications, 2015), pp. 13453-13473.

8. J. E. Yorks, M. J. McGill, V. S. Scott, S. W. Wake, A. Kupchock, D. L. Hlavka, W. D. Hart, and P. A. Selmer, "The Airborne Cloud-Aerosol Transport System: Overview and Description of the Instrument and Retrieval Algorithms," in J Atmos Ocean Tech, (2014), pp. 2482-2497.

9. A. J. Illingworth, H. W. Barker, A. Beljaars, M. Ceccaldi, H. Chepfer, J. Cole, J. Delanoë, C. Domenech, D. P. Donovan, S. Fukuda, M. Hirakata, R. J. Hogan, A. Huenerbein, P. Kollias, T. Kubota, T. Nakajima, T. Y. Nakajima, T. Nishizawa, Y. Ohno, H. Okamoto, R. Oki, K. Sato, M. Satoh, M. Shephard, U. Wandinger, T. Wehr, and G. J. van Zadelhoff, "THE EARTHCARE SATELLITE: The next step forward in global measurements of clouds, aerosols, precipitation and radiation," B Am Meteorol Soc (2015).

10. D. Bruneau, J. Pelon, F. Blouzon, J. Spatazza, P. Genau, G. Buchholtz, N. Amarouche, A. Abchiche, and O. Aouji, "355-nm high spectral resolution airborne lidar LNG: system description and first results," in Appl Optics, (OSA, 2015), pp. 8776-8785.

11. S. T. Seaman, A. L. Cook, S. J. Scola, C. A. Hostetler, I. Miller, and W. Welch, "Performance characterization of a pressure-tuned wide-angle Michelson interferometric spectral filter for high spectral resolution lidar," in SPIE, Lidar Remote Sensing for Environmental Monitoring XV, (2015), pp. 96120H-96120H-96127.

12. A. Ansmann, U. Wandinger, O. Le Rille, D. Lajas, and A. G. Straume, "Particle backscatter and extinction profiling with the spaceborne high- spectral-resolution Doppler lidar ALADIN: methodology and simulations," Appl Optics 46, 6606-6622 (2007).

13. S. J. Scola, J. F. Osmundsen, L. S. Murchison, W. T. Davis, J. M. Fody, C. M. Boyer, A. L. Cook, C. A. Hostetler, S. T. Seaman, I. J. Miller, W. C. Welch, and A. R. Kosmer, "Structural-Thermal-Optical-Performance (STOP) model development and analysis of a field-widened Michelson interferometer," in SPIE Optical Engineering + Applications, (SPIE, 2014), p. 15.

14. R. Gelaro, W. McCarty, M. J. Suárez, R. Todling, A. Molod, L. Takacs, C. A. Randles, A. Darmenov, M. G. Bosilovich, and R. Reichle, "The modernera retrospective analysis for research and applications, version 2 (MERRA-2)," in J Climate, (2017), pp. 5419-5454.

15. A. Behrendt and T. Nakamura, "Calculation of the calibration constant of polarization lidar and its dependency on atmospheric temperature," Optics Express 10, 805-817 (2002).

16. P. Zuidema, J. Redemann, J. Haywood, R. Wood, S. Piketh, M. Hipondoka, and P. Formenti, "Smoke and Clouds above the Southeast Atlantic: Upcoming Field Campaigns Probe Absorbing Aerosol's Impact on Climate," in B Am Meteorol Soc, (2016), pp. 1131-1135.

17. T. Murayama, D. Müller, K. Wada, A. Shimizu, M. Sekiguchi, and T. Tsukamoto, "Characterization of Asian dust and Siberian smoke with multiwavelength Raman lidar over Tokyo, Japan in spring 2003," in Geophys Res Lett, (2004).

18. D. Müller, I. Mattis, U. Wandinger, A. Ansmann, D. Althausen, and A. Stohl, "Raman lidar observations of aged Siberian and Canadian forest fire smoke in the free troposphere over Germany in 2003: Microphysical particle characterization," in J Geophys Res-Atmos, (2005), p. D17201.

19. K. Sassen, "Identifying Atmospheric Aerosols with Polarization Lidar," in Advanced Environmental Monitoring, Y. J. Kim and U. Platt, eds. (Springer-Verlag, Berlin, 2008), pp. 136-142.

20. S. P. Burton, R. A. Ferrare, C. A. Hostetler, J. W. Hair, R. R. Rogers, M. D. Obland, C. F. Butler, A. L. Cook, D. B. Harper, and K. D. Froyd, "Aerosol Classification of Airborne High Spectral Resolution Lidar Measurements Methodology and Examples," in Atmospheric Measurement Techniques, (2012), pp. 73-98.

21. D. Müller, A. Ansmann, I. Mattis, M. Tesche, U. Wandinger, D. Althausen, and G. Pisani, "Aerosol-type-dependent lidar ratios observed with Raman lidar," in J Geophys Res-Atmos, (2007), p. D16202.

22. S. Groß, M. Esselborn, B. Weinzierl, M. Wirth, A. Fix, and A. Petzold, "Aerosol classification by airborne high spectral resolution lidar observations," in Atmos. Chem. Phys., (Copernicus Publications, 2013), pp. 2487-2505.

23. A. Ansmann, H. Baars, M. Tesche, D. Muller, D. Althausen, R. Engelmann, T. Pauliquevis, and P. Artaxo, "Dust and smoke transport from Africa to South America: Lidar profiling over Cape Verde and the Amazon rainforest," in Geophys Res Lett, (2009).

24. M. Tesche, D. Müller, S. Gross, A. Ansmann, D. Althausen, V. Freudenthaler, B. Weinzierl, A. Veira, and A. Petzold, "Optical and microphysical properties of smoke over Cape Verde inferred from multiwavelength lidar measurements," Tellus B 63, 677-694 (2011).

25. E. Giannakaki, P. G. van Zyl, D. Müller, D. Balis, and M. Komppula, "Optical and microphysical characterization of aerosol layers over South Africa by means of multi-wavelength depolarization and Raman lidar measurements," Atmos. Chem. Phys. 16, 8109-8123 (2016).

26. I. Veselovskii, P. Goloub, T. Podvin, D. Tanre, A. da Silva, P. Colarco, P. Castellanos, M. Korenskiy, Q. Hu, D. N. Whiteman, D. Pérez-Ramírez, P. Augustin, M. Fourmentin, and A. Kolgotin, "Characterization of smoke/dust episode over West Africa: comparison of MERRA-2 modeling with multiwavelength Mie-Raman lidar observations," in Atmos. Meas. Tech. Discuss., (Copernicus Publications, 2017), pp. 1-50.

27. D. Nicolae, A. Nemuc, D. Müller, C. Talianu, J. Vasilescu, L. Belegante, and A. Kolgotin, "Characterization of fresh and aged biomass burning events using multiwavelength Raman lidar and mass spectrometry," in Journal of Geophysical Research: Atmospheres, (2013).

28. A. Ångström, "On the Atmospheric Transmission of Sun Radiation and on Dust in the Air," Geografiska Annaler 11, 156-166 (1929).

29. S. P. Burton, E. Chemyakin, X. Liu, K. Knobelspiesse, S. Stamnes, P. Sawamura, R. H. Moore, C. A. Hostetler, and R. A. Ferrare, "Information content and sensitivity of the $3 \beta+2 \alpha$ lidar measurement system for 
aerosol microphysical retrievals," Atmos. Meas. Tech. 9, 5555-5574

(2016).

30. G. Thompson and T. Eidhammer, "A Study of Aerosol Impacts on Clouds and Precipitation Development in a Large Winter Cyclone," J Atmos Sci 71, 3636-3658 (2014).

31. P. E. Saide, G. Thompson, T. Eidhammer, A. M. da Silva, R. B. Pierce, and G. R. Carmichael, "Assessment of biomass burning smoke influence on environmental conditions for multiyear tornado outbreaks by combining aerosol-aware microphysics and fire emission constraints," Journal of Geophysical Research: Atmospheres 121, 10,294-210,311 (2016).

32. J. S. Reid, P. V. Hobbs, R. J. Ferek, D. R. Blake, J. V. Martins, M. R. Dunlap, and C. Liousse, "Physical, chemical, and optical properties of regional hazes dominated by smoke in Brazil," Journal of Geophysical Research: Atmospheres 103, 32059-32080 (1998).

33. C. F. Bohren and D. R. Huffman, eds., Absorption and Scattering of Light by Small Particles (John Wiley, Hoboken, NJ, 1983), p. 530.

34. I. Veselovskii, D. N. Whiteman, A. Kolgotin, E. Andrews, and M. Korenskii, "Demonstration of Aerosol Property Profiling by Multiwavelength Lidar under Varying Relative Humidity Conditions," in J Atmos Ocean Tech, (2009), pp. 1543-1557.

35. D. Müller, C. A. Hostetler, R. A. Ferrare, S. P. Burton, E. Chemyakin, A. Kolgotin, J. W. Hair, A. L. Cook, D. B. Harper, R. R. Rogers, R. W. Hare, C. S. Cleckner, M. D. Obland, J. Tomlinson, L. K. Berg, and B. Schmid, "Airborne Multiwavelength High Spectral Resolution Lidar (HSRL-2) observations during TCAP 2012: vertical profiles of optical and microphysical properties of a smoke/urban haze plume over the northeastern coast of the US," in Atmos. Meas. Tech., (Copernicus Publications, 2014), pp. 3487-3496.

36. C. A. Hostetler, M. J. Behrenfeld, Y. Hu, J. W. Hair, and J. A. Schulien, "Spaceborne Lidar in the Study of Marine Systems," in Annual Review of Marine Science, (2018).

37. NRC, Earth Science and Applications from Space (National Academies of Science Washington, D.C. , 2007). 


\section{Tables}

Table 1. Procedure to assess HSRL-2 contrast ratio for flight data

1. Identify range bins of the cloud-top signal spike in each profile.

2. Integrate the signal returns (calibrated and range corrected) over the spike (a few bins).

3. Extract the atmospheric attenuated molecular signal for the same range interval just above the cloud top; subtract this value from both integrated cloud spike signals.

4. Group the data into segments; further split data from clouds separated by more than $1 \mathrm{~km}$ into separate populations.

5. Do a linear fit on each population and eliminate fits with correlation coefficient $r<0.8$. This eliminates poor fits due to sparse data or very limited dynamic range in the time bin.

6. Smooth over discontinuities and gaps using Hermite polynomial smoothing without extrapolation.

\section{Figure Captions}

Figure 1. The HSRL-2 density-tuned off-axis wide angle Michelson interferometer. Different colors show different light paths through the interferometer, depending on whether the light is reflected or transmitted when first encountering the beam splitter. The off-axis angle is exaggerated in order to show the beam paths clearly.

Figure 2. Interferometer contrast ratios $D / B$ for HSRL-2 flights on board the NASA ER-2 during the 2016 ORACLES deployment.

Figure 3. Shows the error in the aerosol backscatter coefficient when a retrieval is performed with an incorrect contrast ratio. The $x$-axisvalues are positive for inferred contrast ratios larger than the true contrast ratio (over-estimated contrast ratio). The $y$-axis is positive for overestimated backscatter coefficients. Different lines indicate different true contrast ratios $(D / B=20,40$ or 50$)$ and different values of the total scattering ratio (the ratio of total backscattering to molecular backscattering; TSR $=1.5,3,4$, or 11 ), as indicated in the legend.

Figure 4. (a) Shows a simulated extinction profile with gradually changing gradients at both the top and bottom edges of the simulated aerosol profile. The largest gradientalong the top edge is $-0.25 \mathrm{~km}-1$ per $\mathrm{km}$ and occurs at altitude $5 \mathrm{~km}$. The largest gradient along the bottom edge is $0.5 \mathrm{~km}-1$ per $\mathrm{km}$ and occurs at altitude $1 \mathrm{~km}$. (b) Shows the partial derivative of extinction with respect to contrast ratio $(D / B)$. The largest absolute derivatives occur at the locations of the largest gradients in the extinction profile. (c) Shows the error in extinction as a function of error in contrast ratio for the maximum gradient at the bottom edge of the simulated aerosol (gradient $=0.5 \mathrm{~km}-1$ per $\mathrm{km}$ ), and also shows the dependence on total scattering ratio (the profile is scaled to adjust TSR without changing the gradient, to facilitate independently demonstrating the sensitivities).

Figure 5. Shows retrievals at $355 \mathrm{~nm}$ of aerosol backscatter coefficient, aerosol extinction coefficient and lidar ratio (extinction divided by backscatter) for a profile on 18 September 2016 at 11.2 UT for various values of the assumed contrast ratio $D / B$ as given in the legend. Differences in the bottom row are given with respect to the contrast ratio $D / B=48.3$, which is the inferred contrast ratio for this profile, derived using the calibration procedure detailed in Section 5. Lidar data that have been attenuated severely by a thick cloud deck at approximately $1.2 \mathrm{~km}$ have been removed. The scene contains biomass burning aerosol.

Figure 6. Shows the calibrated and range-corrected signal in the particulate-dominated channel (red, thinner line) and the signal in the molecular-dominated channel (blue, thicker line) in the troposphere as a function of range from the instrument (on the NASA ER-2 aircraft), plotted with the range axis reversed so as to match the atmosphere. The data are taken from a flight on 18 September 2016 which surveyed the African biomass burning plume over the SE Atlantic. Vertical resolution is $15 \mathrm{~m}$. The profile time is $14: 00 \mathrm{UT}$ at location $13.79 \mathrm{~S}, 4.39 \mathrm{E}$. The aerosol signal from the African biomass burning plume is visible between range values of about $15.5 \mathrm{~km}$ and $18.2 \mathrm{~km}$ and the signal from the cloud top is the spike at $18.7 \mathrm{~km}$ range. Downrange of the cloud top, the signals in both channels are completely attenuated. The inset box shows the same data zoomed in to show the spike at cloud-top.

Figure 7. Calibrated backscatter lidar data from two cloud layers for a flight of HSRL-2 on 16 September 2016 during ORACLES. The <> notation indicates that the data shown are integrated over a few bins comprising the cloud spike. Data from the particulate-dominated channel is shown on the $x$-axis and data from the molecular-dominated channel is shown on the $y$-axis. Color coding indicates time on an arbitrary scale with blue colors occurring near the start of the flight and red colors occurring near the end of the flight.

Figure 8. A subset of the data shown in Figure 7. Filled circles are used for signals backscattered from a nearly continuous low cloud deck (below $2 \mathrm{~km}$ altitude) and open circles are used for signals backscattered from scattered mid-level clouds (at approximately $5 \mathrm{~km}$ altitude). Note that different time segments have different intercepts and exhibit different dynamic ranges, and that the intercept is correlated with the dynamic range. Color coding indicates time within the selected hour, with blue colors near the beginning of the hour and red colors near the end of the hour.

Figure 9. The time-height cross-section ("curtain") of aerosol backscatter coefficient at $355 \mathrm{~nm}$ on 16 September 2017. A nearly solid opaque cloud deck just above $1 \mathrm{~km}$ can be seen throughout the flight; in many parts of the flight this cloud deck totally attenuates the laser beam so the curtain shows black below the cloud top. Above the stratus cloud deck, the African biomass burning plume is visible up to about $5.2 \mathrm{~km}$. Some partially transparent mid-level clouds (shown in white) are embedded at the top of the smoke layer around 8:45 and 12:20 UT. Red horizontal lines show the four one-hour periods from which the cloud top data is subsetted for Figure 8.

Figure 10. Shows the percent difference in the inferred gain ratio, $\mathrm{gp} / \mathrm{gm}$, if contrastratio $D / B$ is assumed to be 20 in the calculation of gain ratio but is really $X$ (i.e. the contrast ratio plotted on the $\mathrm{x}$-axis). The example shown has $P p / P m$ (signal ratio) $=1.7$, TSR $=1.022$, corresponding to background level of aerosol loading in the free troposphere, where the gain ratio assessment is typically performed.

Figure 11. Profiles of particulate backscatter coefficient, particulate extinction coefficient, particulate depolarization ratio, and lidar ratio at $355 \mathrm{~nm}$ (blue) and $532 \mathrm{~nm}$ (green) plus extinction-related and backscatter-related Ångström exponents for the same two channels. The data are averaged over a 12 minute $(136 \mathrm{~km})$ segment from 11:0211:14 UT on 18 September 2016 (includes the same profile illustrated in Figure 5 and Figure 6) and show smoke transported approximately $240 \mathrm{~km}$ off the coast of Angola. Vertical resolutions are $315 \mathrm{~m}$ for extinction, lidar ratio, and extinction Ångström exponent; and $15 \mathrm{~m}$ for backscatter, particle depolarization ratio, and backscatter-related Ångström exponent. Error bars indicate one standard deviation of the 
variability of the included profiles. Gray shading indicates systematic uncertainty in the $355 \mathrm{~nm}$ channel related to the contrast ratio.

Figure 12. Profiles of particulate backscatter coefficient, particulate extinction coefficient, particulate depolarization ratio, and lidar ratio at $355 \mathrm{~nm}$ (blue) and $532 \mathrm{~nm}$ (green) plus extinction-related and backscatter-related Ångström exponents for the same two channels. Also shown are the relative humidity and aerosol extinction profiles from the WRF model. The data are averaged over a 12-minute segment from 9:54-10:06 UT on 20 September 2016 and show smoke transported off the coast of Africa. Vertical resolutions are $315 \mathrm{~m}$ for extinction, lidar ratio, and extinction Ångström exponent; and $15 \mathrm{~m}$ for backscatter, particle depolarization ratio, and backscatter-related Ångström exponent. Error bars indicate one standard deviation of the variability of the included profiles. Gray shading indicates systematic uncertainty in the $355 \mathrm{~nm}$ channel related to the contrast ratio.

Figure 13. Theoretical results from Mie modeling of various lidar intensive parameters for a monomodal log-normal distribution with varying effective radius. Effective variance is held fixed at 0.195 and refractive index is wavelength independent and held fixed at 1.49i0.01325. In the left panel are shown the lidar ratio at $355 \mathrm{~nm}$ (blue) and $532 \mathrm{~nm}$ (green) and in the right panel are Ångström exponents: extinction-related Ångström exponent between 355 and $532 \mathrm{~nm}$ (black), backscatter-related Ångström exponent between 355 and 532 $\mathrm{nm}$ (blue) and between 532 and $1064 \mathrm{~nm}$ (red).

\section{Figures}



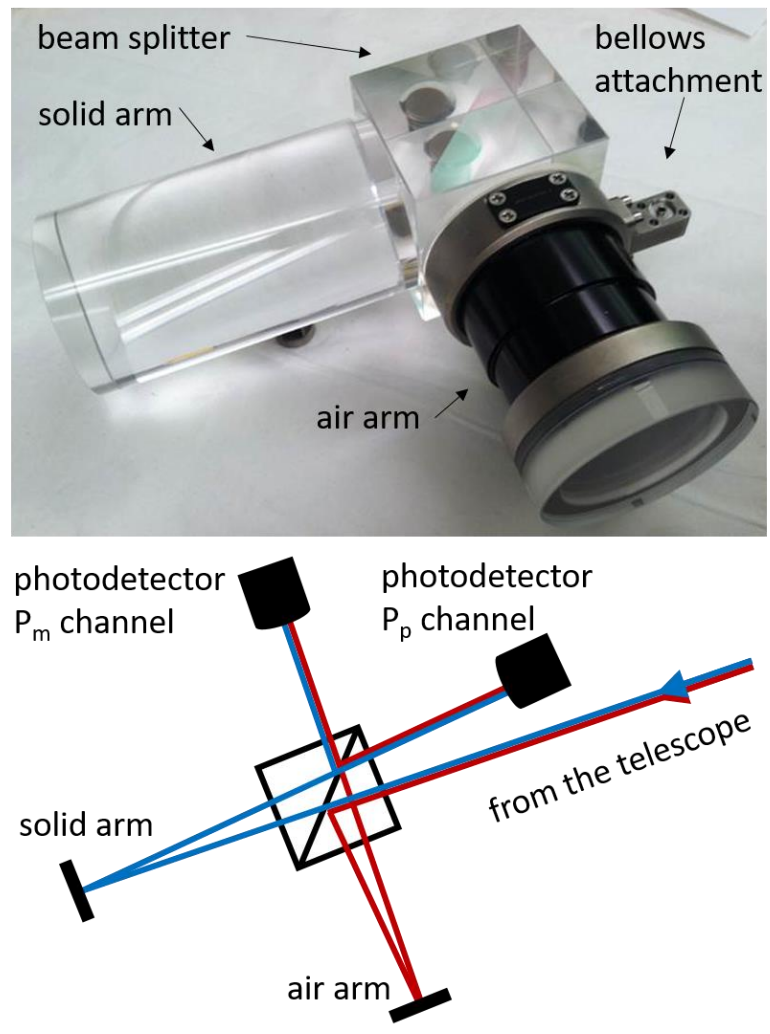

Figure 1. The HSRL-2 density-tuned off-axis wide angle Michelson interferometer. Different colors show different light paths through the interferometer, depending on whether the light is reflected or transmitted when first encountering the beam splitter. The off-axis angle is exaggerated in order to show the beam paths clearly. 


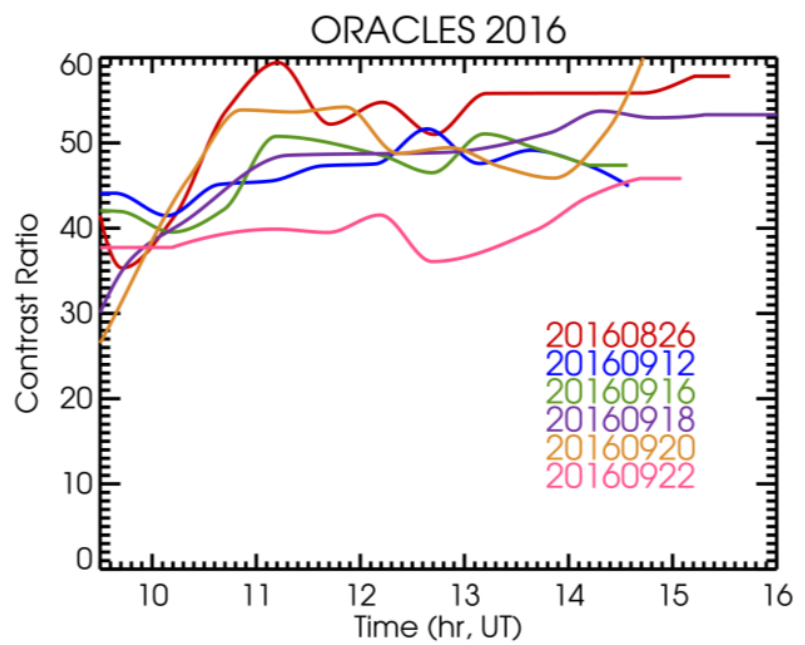

Figure 2. Interferometer contrast ratios $D / B$ for HSRL-2 flights on board the NASA ER-2 during the 2016 ORACLES deployment. 


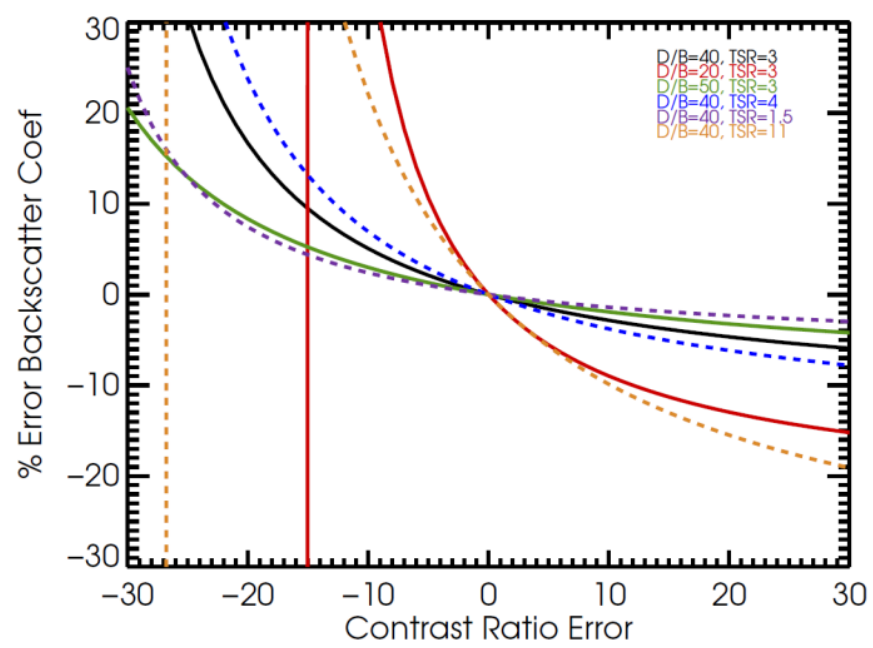

Figure 3. Shows the error in the aerosol backscatter coefficient when a retrieval is performed with an incorrect contrast ratio. The $x$-axis values are positive for inferred contrast ratios larger than the true contrast ratio (over-estimated contrast ratio). The $y$-axis is positive for over-estimated backscatter coefficients. Different lines indicate different true contrast ratios $(D / B=20,40$ or 50$)$ and different values of the total scattering ratio (the ratio of total backscattering to molecular backscattering; $\mathrm{TSR}=1.5,3$, 4 , or 11), as indicated in the legend. 

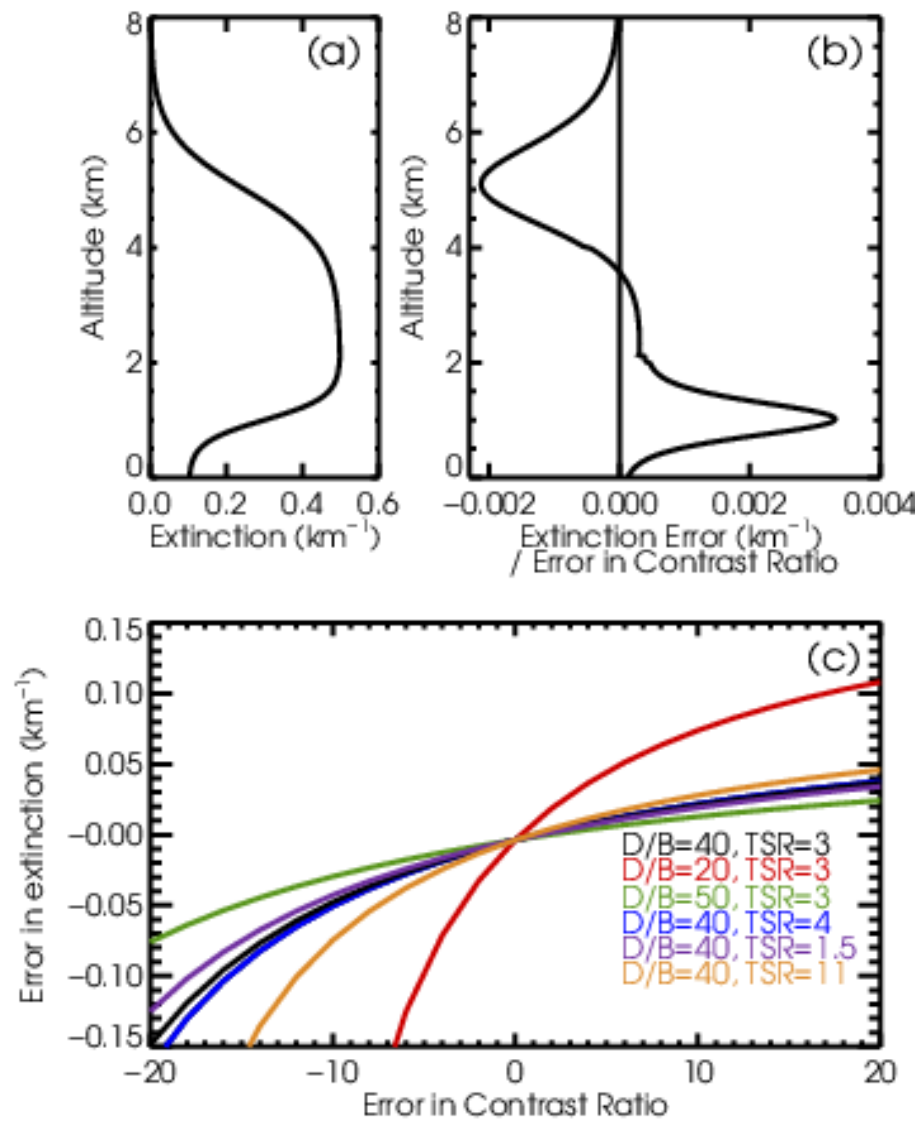

Figure 4. (a) Shows a simulated extinction profile with gradually changing gradients at both the top and bottom edges of the simulated aerosol profile. The largest gradient along the top edge is $-0.25 \mathrm{~km}^{-1}$ per km and occurs at altitude $5 \mathrm{~km}$. The largest gradient along the bottom edge is $0.5 \mathrm{~km}^{-1}$ per km and occurs at altitude $1 \mathrm{~km}$. (b) Shows the partial derivative of extinction with respect to contrast ratio $(D / B)$. The largest absolute derivatives occur at the locations of the largestgradients in the extinction profile. (c) Shows the error in extinction as a function of error in contrast ratio for the maximum gradient at the bottom edge of the simulated aerosol (gradient $=0.5 \mathrm{~km}^{-1}$ per $\mathrm{km}$ ), and also shows the dependence on total scattering ratio (the profile is scaled to adjust TSR without changing the gradient, to facilitate independently demonstrating the sensitivities). 

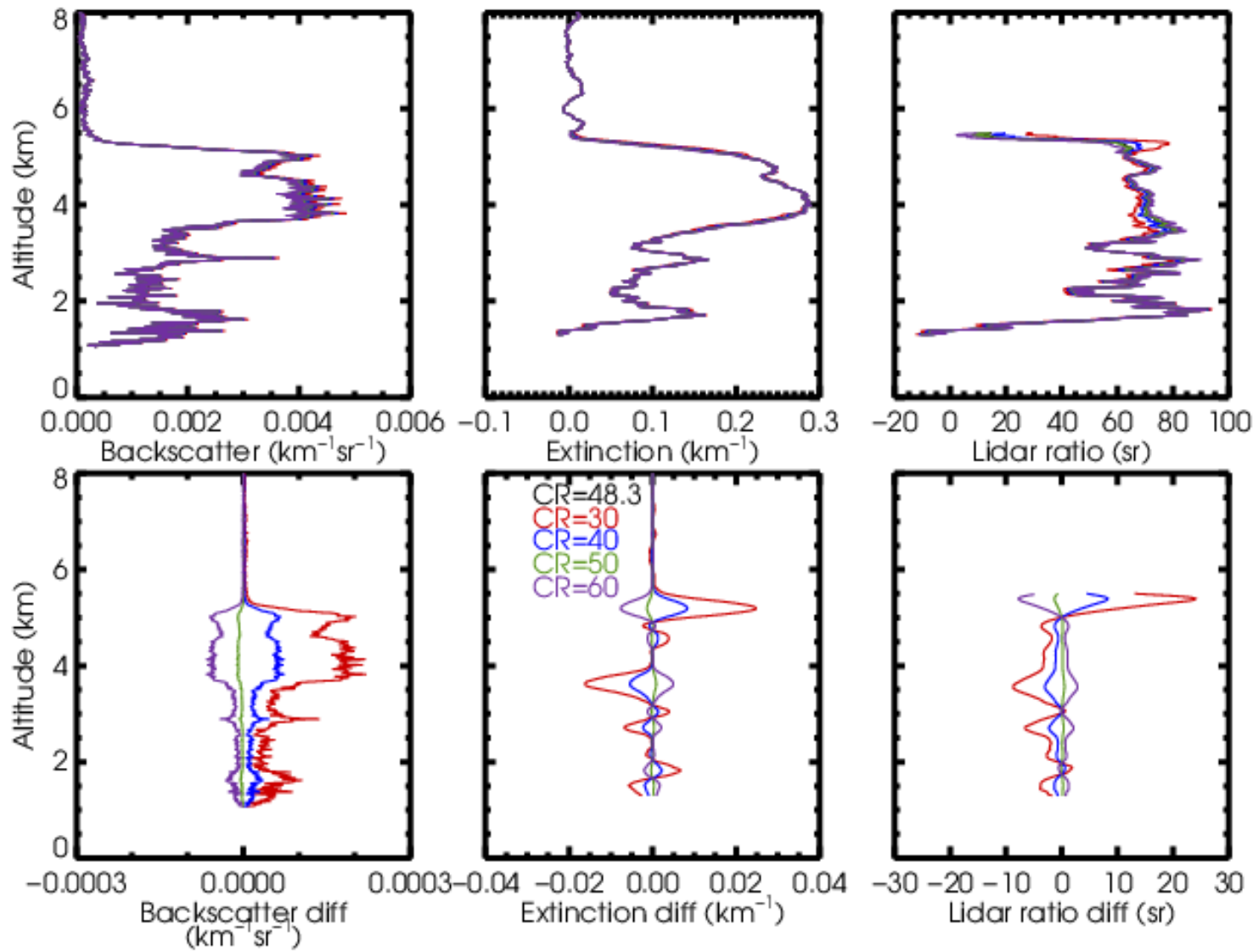

Figure 5. Shows retrievals at $355 \mathrm{~nm}$ of aerosol backscatter coefficient, aerosol extinction coefficient and lidar ratio (extinction divided by backscatter) for a profile on 18September 2016 at 11.2 UT for various values of the assumed contrast ratio $D / B$ as given in the legend. Differences in the bottom row are given with respect to the contrast ratio $D / B=48.3$, which is the inferred contrast ratio for this profile, derived using the calibration procedure detailed in Section 5. Lidar data that have been attenuated severely by a thick cloud deck at approximately $1.2 \mathrm{~km}$ have been removed. The scene contains biomass burning aerosol. 


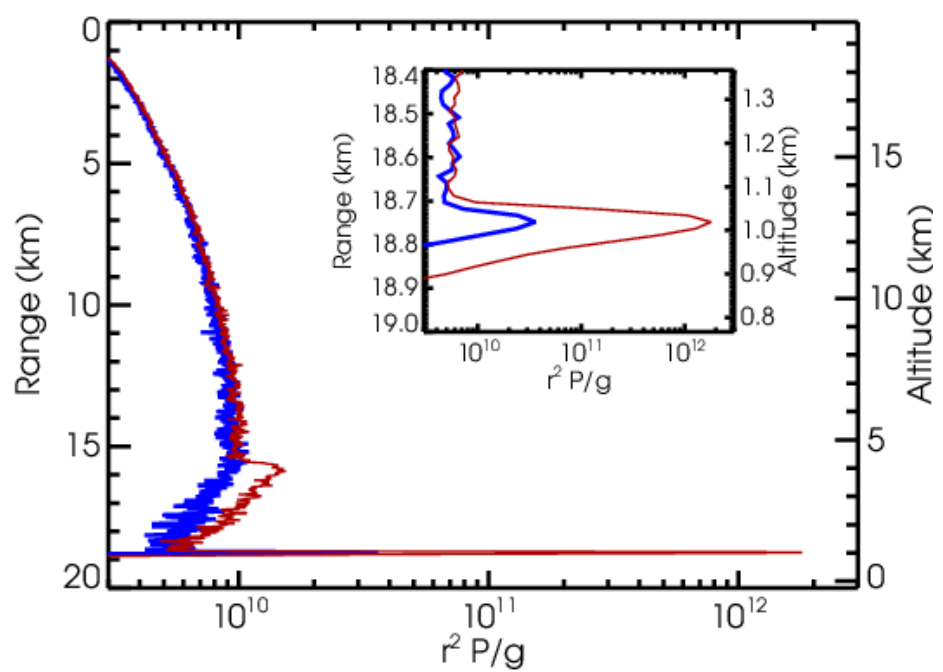

Figure 6. Shows the calibrated and range-corrected signal in the particulatedominated channel (red, thinner line) and the signal in the moleculardominated channel (blue, thicker line) in the troposphere as a function of range from the instrument (on the NASA ER-2 aircraft), plotted with the range axis reversed so as to match the atmosphere. The data are taken from a flight on 18 September 2016 which surveyed the African biomass burning plume over the SE Atlantic. Vertical resolution is $15 \mathrm{~m}$. The profile time is 14:00 UT at location 13.79S, 4.39 E. The aerosol signal from the African biomass burning plume is visible between range values of about $15.5 \mathrm{~km}$ and $18.2 \mathrm{~km}$ and the signal from the cloud top is the spike at $18.7 \mathrm{~km}$ range. Downrange of the cloud top, the signals in both channels are completely attenuated. The inset box shows the same data zoomed in to show the spike at cloud-top. 


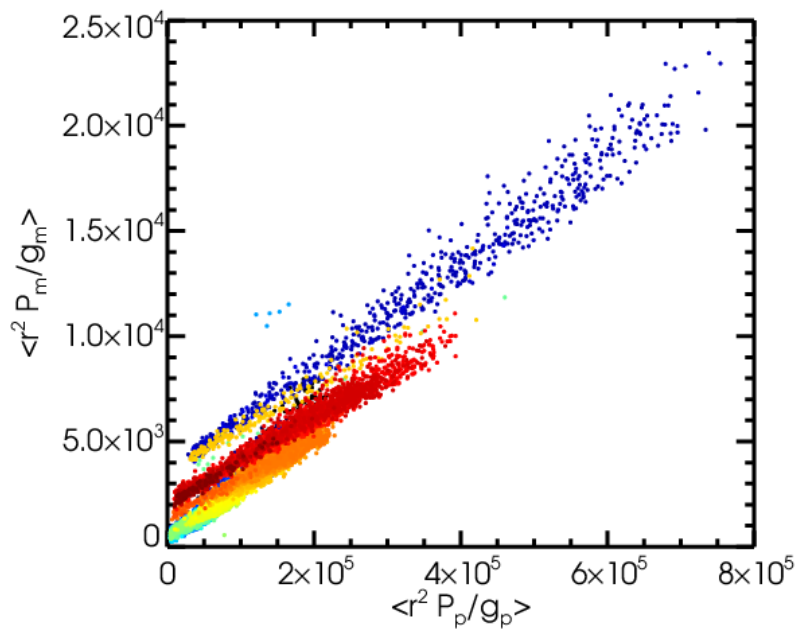

Figure 7. Calibrated backscatter lidar data from two cloud layers for a flight of HSRL-2 on 16 September 2016 during ORACLES. The <> notation indicates that the data shown are integrated over a few bins comprising the cloud spike. Data from the particulate-dominated channel is shown on the $x$-axis and data from the moleculardominated channel is shown on the $y$-axis. Color coding indicates time on an arbitrary scale with blue colors occurring near the start of the flight and red colors occurring near the end of the flight. 


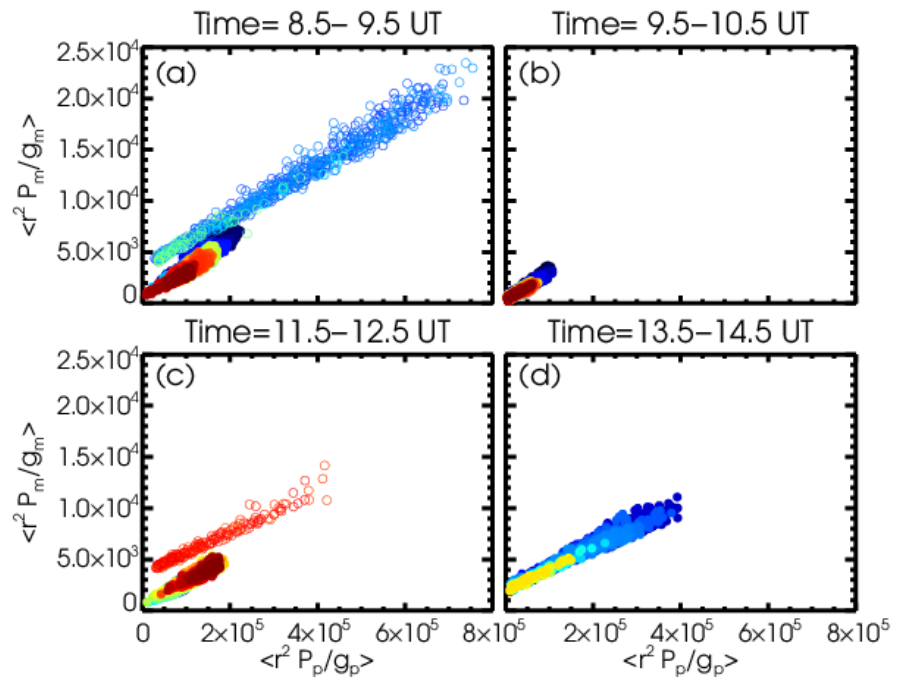

Figure 8. A subset of the data shown in Figure 7. Filled circles are used for signals backscattered from a nearly continuous low cloud deck (below $2 \mathrm{~km}$ altitude) and open circles are used for signals backscattered from scattered mid-level clouds (at approximately $5 \mathrm{~km}$ altitude). Note that different time segments have different intercepts and exhibit different dynamic ranges, and that the intercept is correlated with the dynamic range. Color coding indicates time within the selected hour, with blue colors near the beginning of the hour and red colors near the end of the hour. 


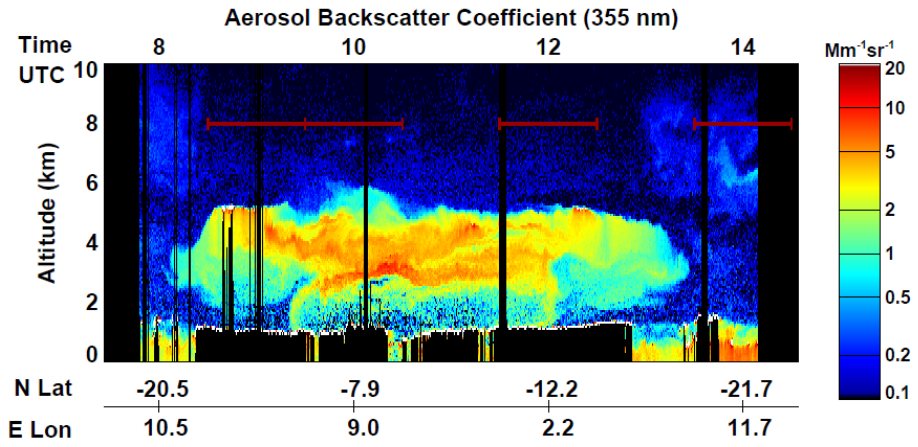

Figure 9. The time-height cross-section ("curtain") of aerosol backscatter coefficient at $355 \mathrm{~nm}$ on 16 September 2017. A nearly solid opaque cloud deck just above $1 \mathrm{~km}$ can be seen throughout the flight; in many parts of the flight this cloud deck totally attenuates the laser beam so the curtain shows black below the cloud top. Above the stratus cloud deck, the African biomass burning plume is visible up to about $5.2 \mathrm{~km}$. Some partially transparent midlevel clouds (shown in white) are embedded at the top of the smoke layer around 8:45 and 12:20 UT. Red horizontal lines show the four one-hour periods from which the cloud top data is subsetted for Figure 8. 


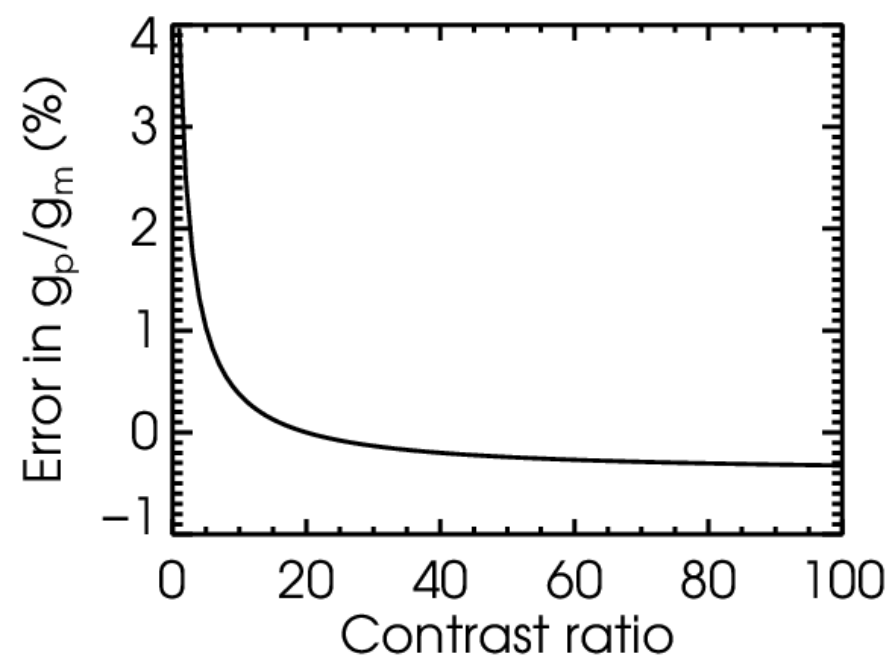

Figure 10. Shows the percent difference in the inferred gain ratio, $g_{p} / g_{m}$, if contrast ratio $D / B$ is assumed to be 20 in the calculation of gain ratio but is really $X$ (i.e. the contrastratio plotted on the $\mathrm{x}$-axis). The example shown has $P_{p} / P_{m}$ (signal ratio) $=1.7, \mathrm{TSR}=1.022$, corresponding to background level of aerosol loading in the free troposphere, where the gain ratio assessment is typically performed. 

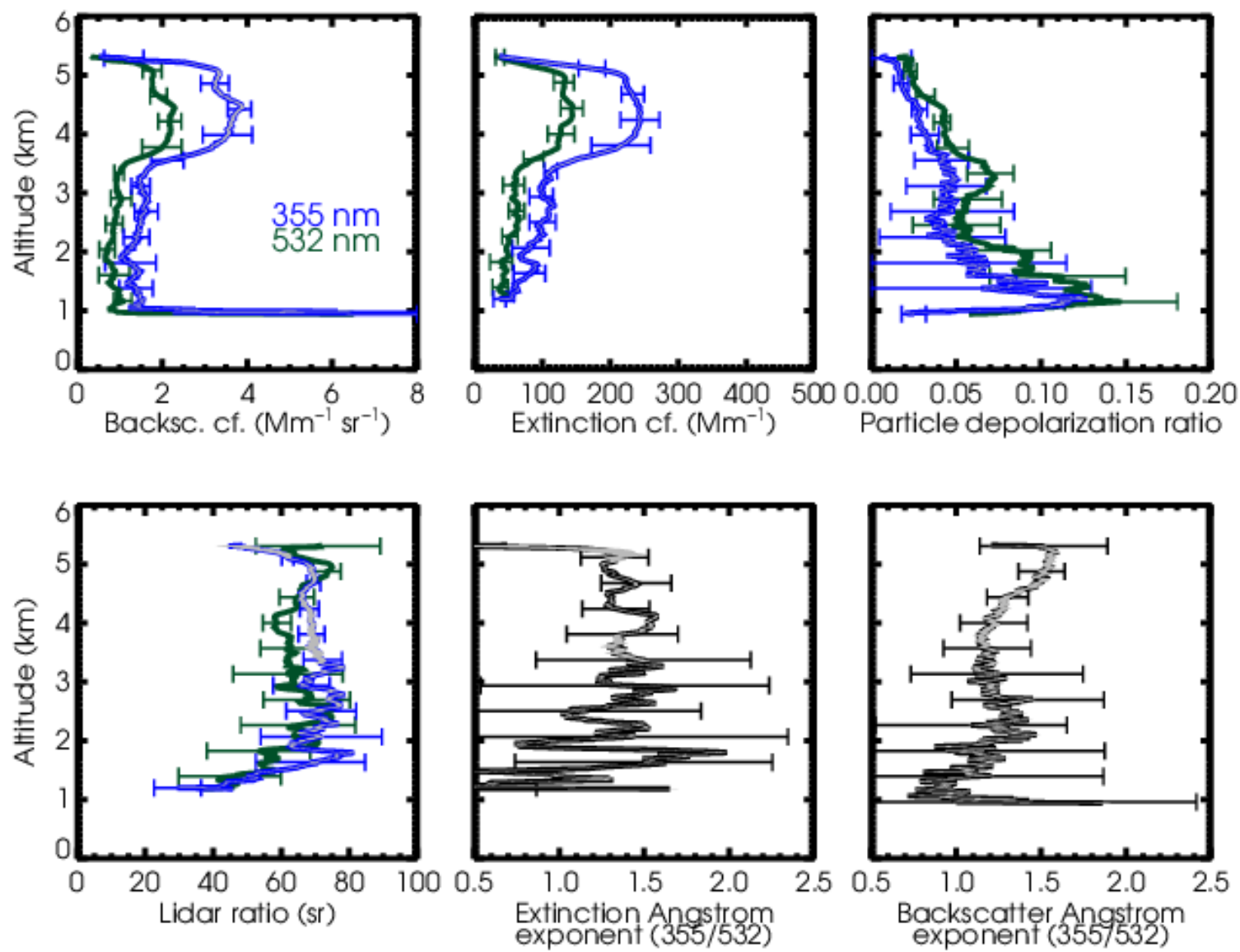

Figure 11. Profiles of particulate backscatter coefficient, particulate extinction coefficient, particulate depolarization ratio, and lidar ratio at $355 \mathrm{~nm}$ (blue) and $532 \mathrm{~nm}$ (green) plus extinction-related and backscatter-related Ångström exponents for the same two channels. The data are averaged over a 12 minute $(136 \mathrm{~km})$ segment from 11:02-11:14 UT on 18 September 2016 (includes the same profile illustrated in Figure 5 and Figure 6) and show smoke transported approximately $240 \mathrm{~km}$ off the coast of Angola. Vertical resolutions are $315 \mathrm{~m}$ for extinction, lidar ratio, and extinction Ångström exponent; and $15 \mathrm{~m}$ for backscatter, particle depolarization ratio, and backscatter-related Ångström exponent. Error bars indicate one standard deviation of the variability of the included profiles. Gray shading indicates systematic uncertainty in the $355 \mathrm{~nm}$ channel related to the contrast ratio. 

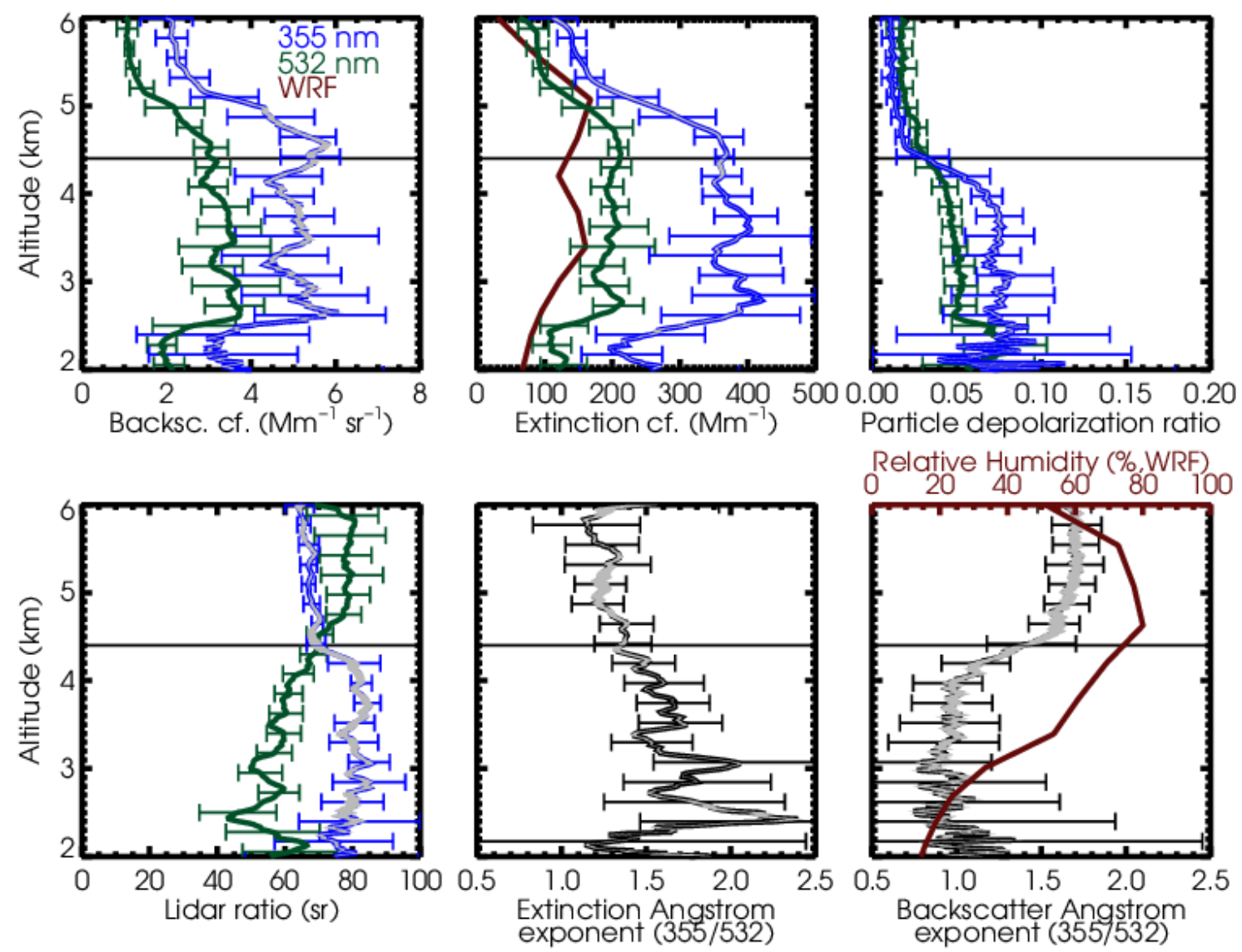

Figure 12. Profiles of particulate backscatter coefficient, particulate extinction coefficient, particulate depolarization ratio, and lidar ratio at 355 $\mathrm{nm}$ (blue) and $532 \mathrm{~nm}$ (green) plus extinction-related and backscatter-related Ångström exponents for the same two channels. Also shown are the relative humidity and aerosol extinction profiles from the WRF model. The data are averaged over a 12-minute segment from 9:54-10:06 UT on 20 September 2016 and show smoke transported off the coast of Africa. Vertical resolutions are 315 m for extinction, lidar ratio, and extinction Ångström exponent; and 15 m for backscatter, particle depolarization ratio, and backscatter-related Ångström exponent. Error bars indicate one standard deviation of the variability of the included profiles. Gray shading indicates systematic uncertainty in the $355 \mathrm{~nm}$ channel related to the contrast ratio. 

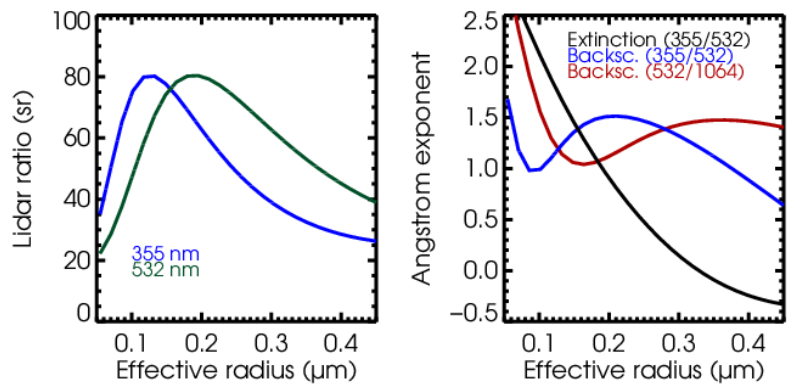

Figure 13. Theoretical results from Mie modeling of various lidar intensive parameters for a monomodal log-normal distribution with varying effective radius. Effective variance is held fixed at 0.195 and refractive index is wavelength independent and held fixed at 1.49-i0.01325. In the left panel are shown the lidar ratio at $355 \mathrm{~nm}$ (blue) and $532 \mathrm{~nm}$ (green) and in the right panel are Ångström exponents: extinction-related Ångström exponent between 355 and $532 \mathrm{~nm}$ (black), backscatter-related Ångström exponent between 355 and $532 \mathrm{~nm}$ (blue) and between 532 and $1064 \mathrm{~nm}$ (red). 